\title{
Construction and Response of a Highly Granular Scintillator-based Electromagnetic Calorimeter
}

\author{
The CALICE Collaboration*
}

\begin{abstract}
A highly granular electromagnetic calorimeter with scintillator strip readout is being developed for future lepton collider experiments. A prototype of $21.5 X_{0}$ depth and $180 \times 180 \mathrm{~mm}^{2}$ transverse dimensions was constructed, consisting of 2160 individually read out $10 \times 45 \times 3 \mathrm{~mm}^{3}$ scintillator strips. This prototype was tested using electrons of $2-32 \mathrm{GeV}$ at the Fermilab Test Beam Facility in 2009. Deviations from linear energy response were less than 1.1\%, and the intrinsic energy resolution was determined to be $(12.6 \pm 0.1$ (stat.) \pm 0.4 (syst.) $) \% / \sqrt{E[\mathrm{GeV}]} \oplus$ $(1.1 \pm 0.1 \text { (stat. })_{-0.7}^{+0.6}$ (syst.) $) \%$, where the uncertainties correspond to statistical and systematic sources, respectively.
\end{abstract}

${ }^{*}$ Corresponding author: Katsushige Kotera, (coterra@champ.hep.sci.osaka-u.ac.jp) 


\title{
The CALICE Collaboration
}

\author{
J. Repond, L. Xia \\ Argonne National Laboratory, 9700 S. Cass Avenue, Argonne, IL 60439-4815, USA \\ G. Eigen, \\ University of Bergen, Inst. of Physics, Allegaten 55, N-5007 Bergen, Norway
}

T. Price, N.K. Watson, A. Winter

University of Birmingham, School of Physics and Astronomy, Edgbaston, Birmingham B15

2TT, UK

M.A. Thomson,

University of Cambridge, Cavendish Laboratory, J J Thomson Avenue, CB3 0HE, UK

G. C. Blazey, A. Dyshkant, K. Francis, V. Zutshi

NICADD, Northern Illinois University, Department of Physics, DeKalb, IL 60115, USA

K. Gadow, P. Göttlicher, O. Hartbrich包, K. Kotera, F. Krivan, K. Krüger, S. Lu,

B. Lutz, M. Reinecke, F. Sefkow, Y.Sudo, H.L. Tran

DESY, Notkestrasse 85, D-22603 Hamburg, Germany

A. Kaplan,H.-Ch. Schultz-Coulon,

University of Heidelberg, Fakultat fur Physik und Astronomie, Albert Uberle Str. 3-5 2.OG

Ost, D-69120 Heidelberg, Germany

B. Bilki, D. Northacker, Y. Onel

University of Iowa, Dept. of Physics and Astronomy, 203 Van Allen Hall, Iowa City, IA 52242-1479, USA

G.W. Wilson

University of Kansas, Department of Physics and Astronomy, Malott Hall, 1251 Wescoe Hall Drive, Lawrence, KS 66045-7582, USA

K. Kawagoe, I. Sekiya, T. Suehara, H. Yamashiro, T. Yoshioka,

Department of Physics and Research Center for Advanced Particle Physics, Kyushu University, 744 Motooka, Nishi-ku, Fukuoka 819-0395, Japan

E. Calvo Alamillo, M.C. Fouz, J. Marin, J. Navarrete, J. Puerta Pelayo, A. Verdugo

CIEMAT, Centro de Investigaciones Energeticas, Medioambientales y Tecnologicas, Madrid, Spain

\section{Chadeevad, M. Danilove, \\ P. N. Lebedev Physical Institute, Russian Academy of Sciences, 117924 GSP-1 Moscow, B-333, Russia}

\section{Gabriel, P. Goecke, C. Graf, Y. Israeli, N. van der Kolk, F. Simon, M. Szalay, H. Windel \\ Max-Planck-Institut für Physik, Föhringer Ring 6, D-80805 Munich, Germany}

\footnotetext{
${ }^{a}$ now at University of Hawaii at Manoa, High Energy Physics Group, 2505 Correa Road, HI, Honolulu 96822, USA

balso at Shinshu University, now at Osaka University

${ }^{\mathrm{c}}$ Also at Beykent University, Istanbul, Turkey

${ }^{\mathrm{d}}$ Also at MEPhI

${ }^{\mathrm{e}} \mathrm{Also}$ at MEPhI and at Moscow Institute of Physics and Technology
} 
S. Bilokin, J. Bonis, R. Pöschl, A. Thiebault, F. Richard, D. Zerwas Laboratoire de l'Accélerateur Linéaire, CNRS/IN2P3 et Université de Paris-Sud XI, Centre Scientifique d'Orsay Bâtiment 200, BP 34, F-91898 Orsay CEDEX, France

M. Anduze, V. Balagura, V. Boudry, J-C. Brient, R. Cornat, Laboratoire Leprince-Ringuet (LLR) - École Polytechnique, CNRS/IN2P3, Palaiseau, F-91128 France

J. Cvach, M. Janata, M. Kovalcuk, J. Kvasnickaf, I. Polak, J. Smolik, V. Vrba, J. Zalesak, J. Zuklin

Institute of Physics, The Czech Academy of Sciences, Na Slovance 2, CZ-18221 Prague 8, Czech Republic

W. Choi, K. Koterag, M. Nishiyama, T. Sakuma, T. Takeshita, S. Tozuka, T. Tsubokawa, S. Uozumin,

Shinshu Univ., Dept. of Physics, 3-1-1, Asahi, Matsumoto-shi, Nagano 390-8621, Japan

D. Jeans, W. Ootani, L. Liu, ICEPP, The University of Tokyo, 7-3-1 Hongo, Bunkyo-ku, Tokyo 113-0033, Japan

S. Chang, A. Khanj, D.H. Kim, D.J. Kong, Y.D. Oh

Department of Physics, Kyungpook National University, Daegu, 702-701, Republic of Korea

T. Ikuno, Y.Sudo, Y. Takahashi

Division of Physics, Faculty of Pure and Applied Sciences, University of Tsukuba, Tennoudai 1-1-1, Tsukuba-shi, Ibaraki-ken 305-8571, Japan

M. Götze,

Bergische Universität Wuppertal Fakultät 4 / Physik, Gaussstrasse 20, D-42097 Wuppertal, Germany

\footnotetext{
${ }^{f}$ also at DESY

${ }^{\mathrm{g}}$ also at DESY, now at Osaka University

${ }^{\mathrm{h}}$ now at Okayama University

inow at KEK

${ }^{\mathrm{j}}$ now at Islamia College University, Peshawar, Pakistan
} 


\section{Contents}

1 Introduction

2 Construction 5

2.1 Detector .......................... 5

2.2 Data acquisition system . . . . . . . . . . . . . . . . . . 9

2.3 Characterisation of MPPCs and their non-linear response . . . . . . . . . . . 9

3 Test beam at FNAL 10

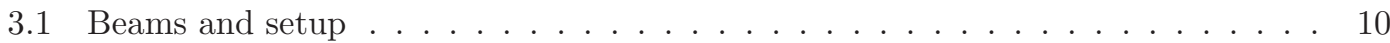

3.2 Temperature measurement . . . . . . . . . . . . . . . . . . 13

4 Reconstruction procedure 14

4.1 Calibration procedure . . . . . . . . . . . . . . . . 14

4.2 Calibration runs and pedestal measurements . . . . . . . . . . . . . 15

4.3 Determination of calibration constants . . . . . . . . . . . . . . . . . 15

4.3.1 ADC-MIP conversion factor . . . . . . . . . . . . . . . . . . 15

4.3.2 ADC-photoelectron conversion factor . . . . . . . . . . . . . . 16

4.3 .3 Inter-calibration constant . . . . . . . . . . . . . . . . . . 18

4.4 Reconstruction of electron energy spectra . . . . . . . . . . . . . 19

5 Performance of the prototype 22

5.1 Mean and resolution with statistical uncertainties . . . . . . . . . . . . . 22

5.2 Systematic uncertainties . . . . . . . . . . . . . . . . . . 22

5.3 Linearity and energy resolution of the ScECAL prototype . . . . . . . 26

6 Comparison with Monte Carlo simulation 29

6.1 ScECAL prototype simulation . . . . . . . . . . . . . . . 29

6.2 Shower profile. . . . . . . . . . . . . . . . . . . . . 31

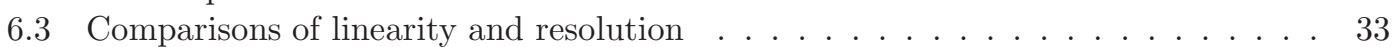

7 Discussion 33

8 Conclusion 36

9 Acknowledgement 36

A Composition of the absorber plate 


\section{Introduction}

Detectors for the International Linear Collider (ILC) are designed to perform high precision measurements, taking advantage of the well-defined initial conditions of electron-positron collisions. To characterise final states that are dominated by the production and decay of quarks, gauge bosons and/or Higgs bosons, the accurate reconstruction of jets of hadrons is mandatory. One way to achieve this is by measuring each particle within a jet individually, and combining information from calorimeters and tracking detectors. This method, known as the particle flow approach (PFA) [1,2], requires highly granular calorimeters. The requirement of single particle separation within a jet translates for the electromagnetic calorimeter (ECAL) in a lateral segmentation better then the Molière radius of Tungsten $(9.3 \mathrm{~mm})$, and a longitudinal sampling at least every $X_{0}$, resulting in 20-30 layers. At the ILC, an ideal value for the intrinsic energy resolution of the ECAL is required to be less than $15 \% / \sqrt{E[\mathrm{GeV}]}$ by PFA 2 . Emerging designs for scintillator-based sampling calorimeters now have the potential to realise these design criteria.

The previous limiting factors for the segmentation of a scintillator-based calorimeter were the size and sensitivity of the readout technology. This situation changed drastically with the introduction of silicon photomultiplier (SiPM) 4 8. Small scintillator elements can be read out individually using SiPMs without introducing large dead volumes for the readout systems. This technology is used in the scintillator strip electromagnetic calorimeter (ScECAL) being developed by the CALICE Collaboration. To reduce both the total number of readout channels and the overall insensitive volume associated with the readout SiPMs, strips of scintillator, each with a length of $45 \mathrm{~mm}$ and a width of between 5 and $10 \mathrm{~mm}$, are used. Strips in successive layers have an orthogonal orientation relative to each other 9] and an algorithm has been developed to achieve fine effective segmentation from such a strip-based design [10. To achieve the required longitudinal segmentation, the ScECAL is designed as a sampling calorimeter using 25-30 tungsten layers of thickness of 2-4 mm, interleaved with scintillator strip sensor layers. The first CALICE ScECAL prototype 11] consisted of 26 sensor layers, interleaved with $3.5 \mathrm{~mm}$ thick tungsten carbide (WC) absorber layers, and had a transverse area of $90 \times 90 \mathrm{~mm}^{2}$.

The current prototype consists of 30 detector layers and has transverse dimensions of $180 \times$ $180 \mathrm{~mm}^{2}$ and a depth of $21.5 X_{0}(266 \mathrm{~mm})$, reducing the effect of lateral and longitudinal shower leakage relative to the previous prototype. The basic unit was a $45 \times 10 \times 3 \mathrm{~mm}^{3}$ scintillator strip with a central hole of $1.5 \mathrm{~mm}$ diameter running along its length, hermetically wrapped with reflective foil. A wavelength shifting (WLS) fibre inserted into the hole guides light to a SiPM placed at one of the ends of the scintillator strip. A LED-based gain monitoring system was implemented for each strip, an improvement on the first prototype in which only one LED was provided per layer. This prototype was tested in conjunction with the CALICE analogue hadron calorimeter (AHCAL) [7, 12, 1] and tail catcher muon tracker (TCMT) [13, prototypes.

This paper is organised as follows. Details of the prototype design including properties of applied SiPMs are given in Section2. The test beam experiment at Fermilab is described in Section 3, and the analysis including detector calibration and results obtained using electron beams are given in Sections 4 and 5. Section 6 compares the analysis results with Monte Carlo simulations, Section 7 discusses the results and Section 8 draws conclusions.

\section{Construction}

\subsection{Detector}

The prototype, shown in Fig. 1 in front of the CALICE AHCAL, has a total thickness of $266 \mathrm{~mm}$. It consists of 30 pairs of alternating $3.5 \mathrm{~mm}$ thick tungsten carbide absorber 


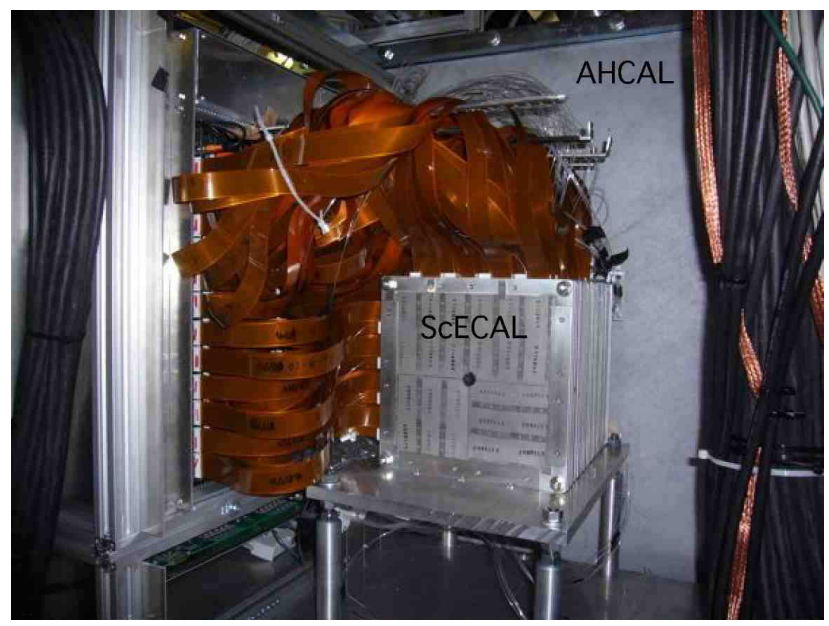

Figure 1: The ScECAL prototype in front of the CALICE AHCAL.

and scintillator layers, with the first layer being absorber. Figure 2 shows the design of a scintillator layer, consisting of four rows of 18 scintillator strips, held in a rigid steel frame. Figure 3 illustrates the design of a single polystyrene-based scintillator strip and shows the central hole for the WLS fibre, manufactured using an extrusion method [14 and cut into strips. The polystyrene was doped using a mixture of $1 \% 2,5$-diphenyloxazole and $0.1 \% 2,2$ '( $p$-phenylene)bis(5-phenyloxazole) for fluorescence. A notch with a depth of $1.40 \pm 0.05 \mathrm{~mm}$ and a width of $4.46 \pm 0.03 \mathrm{~mm}$ was cut mechanically to accommodate the SiPM. The specific SiPM used was a multi-pixel photon counter (MPPC), from Hamamatsu K.K. 15. The size of the MPPC package was $1.3 \times(4.2 \pm 0.2) \times(3.2 \pm 0.2) \mathrm{mm}^{3}$. The four long sides of each strip were polished to control precisely the strip size and to ensure reflection of the surfaces.

From a randomly chosen sample of 20 strips, the measured mean values and the sample standard deviations (SD) of the widths, lengths and thicknesses were $9.85 \pm 0.01 \mathrm{~mm}, 44.71 \pm$ $0.04 \mathrm{~mm}$, and $3.02 \pm 0.02 \mathrm{~mm}$, respectively. A double clad $1 \mathrm{~mm}$ diameter Y-11 WLS fibre provided by KURARAY Co., Ltd. [16] with a length of $43.6 \pm 0.1 \mathrm{~mm}$ was inserted into the hole of each strip. Each strip was wrapped with a $57 \mu \mathrm{m}$-thick reflective foil provided by KIMOTO Co., Ltd [17. This foil consists of layers of silver and aluminium, deposited by evaporation between layers of polyethylene terephthalate, and has a reflection ratio of $95.2 \%$ for light with a wavelength of $450 \mathrm{~nm}$. Four out of 2160 channels of the present ScECAL prototype were not operational. One possible cause is the development of short-circuits between the MPPC electrodes caused by the conductive cut edges of the reflector film. The CALICE Collaboration has another candidate for the reflector design that does not have any conductive layer [18. Each scintillator strip also has a $2.5 \mathrm{~mm}$ diameter hole in the reflective foil to allow the injection of light from a LED for gain monitoring.

A screen, also made of reflective foil, was used to prevent scintillation photons impinging directly onto the MPPC, without passing through the WLS fibre, to ensure uniformity of response along the length of the strip. When the screen is used, the response to single particles at the end of the strip furthest from the MPPC is $(88.3 \pm 0.4) \%$ of that directly in front of the MPPC. This is discussed in more detail in Section 7. A photograph of the screen attached to the inside of the scintillator notch is shown in Fig. 4. Nine MPPCs were soldered onto a flat

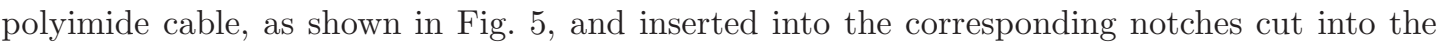
scintillator strips.

Each pair of absorber and scintillator layers was held in a rigid steel frame. Each frame held four $100 \times 100 \times(3.49 \pm 0.01) \mathrm{mm}^{3}$ tungsten carbide plates aligned to make a $200 \times 200 \mathrm{~mm}^{2}$ absorber layer in front of the scintillator. The density of the absorber plates, based on a

\footnotetext{
${ }^{1}$ Electromagnetic response of AHCAL is also available.
} 


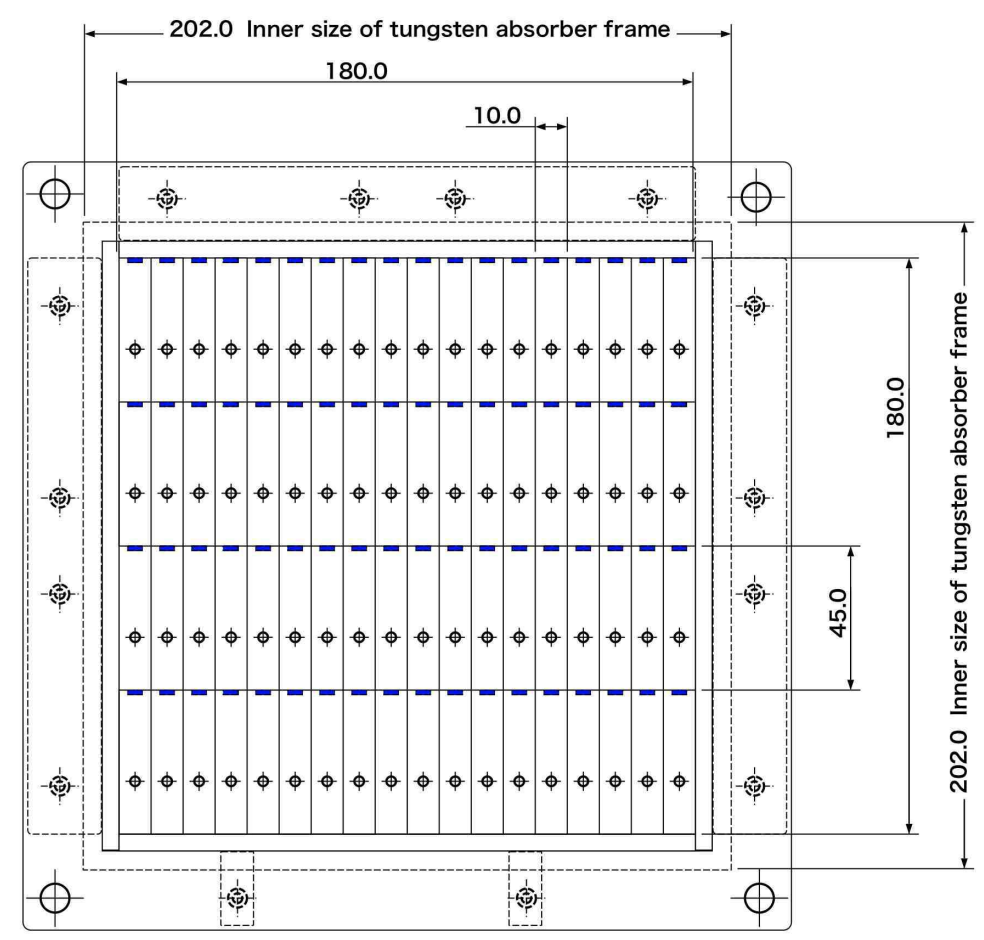

Figure 2: The arrangement of 72 strips in a scintillator layer. The positions of the MPPC housing notches (blue) are shown, as well as the holes in the reflector foil used for the LED calibration. All dimensions are given in $\mathrm{mm}$.
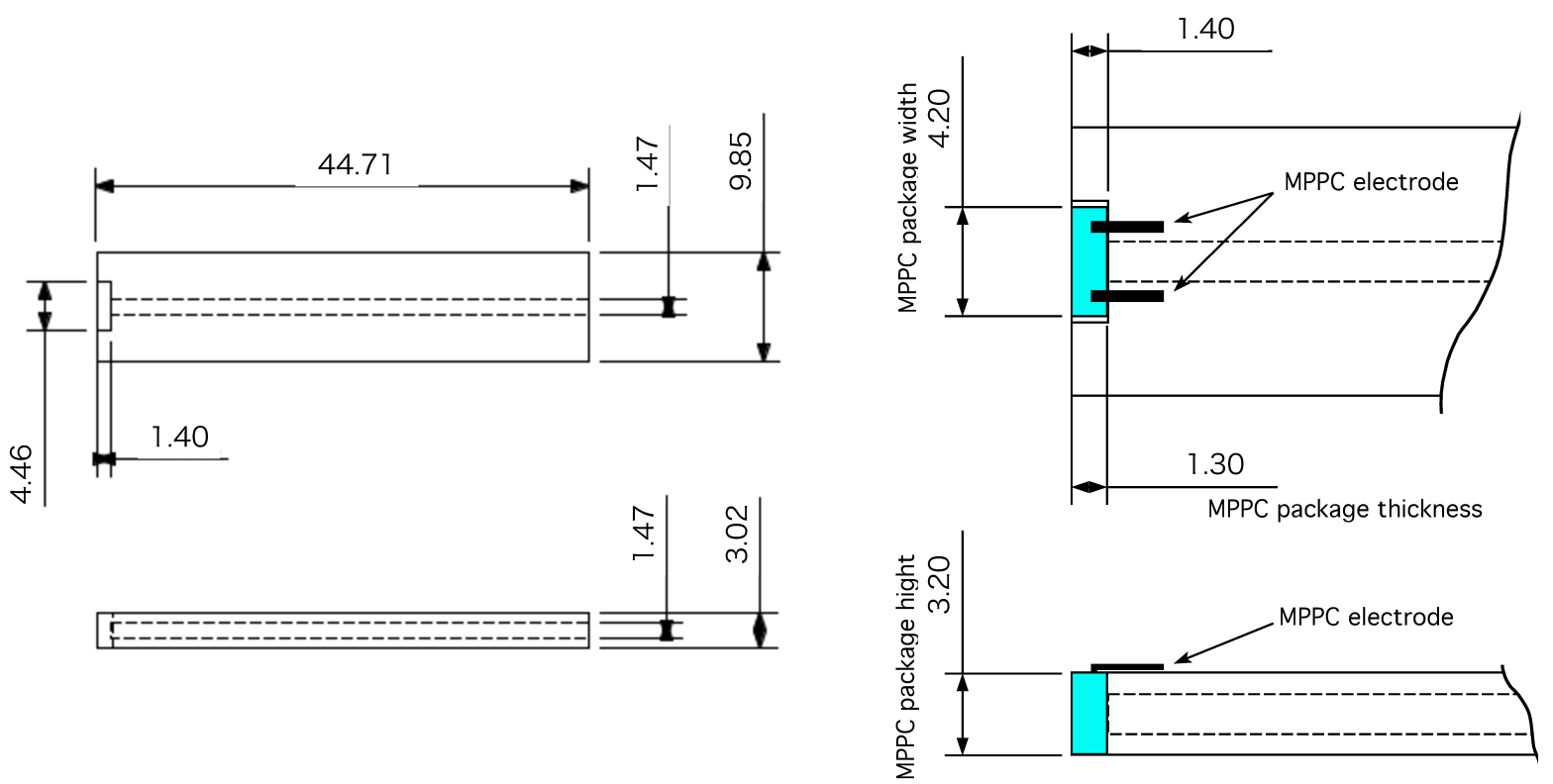

Figure 3: Top and side views of a scintillator strip (left) and the notches cut into the strips to accommodate the MPPC packages (right). All dimensions are given in $\mathrm{mm}$. 


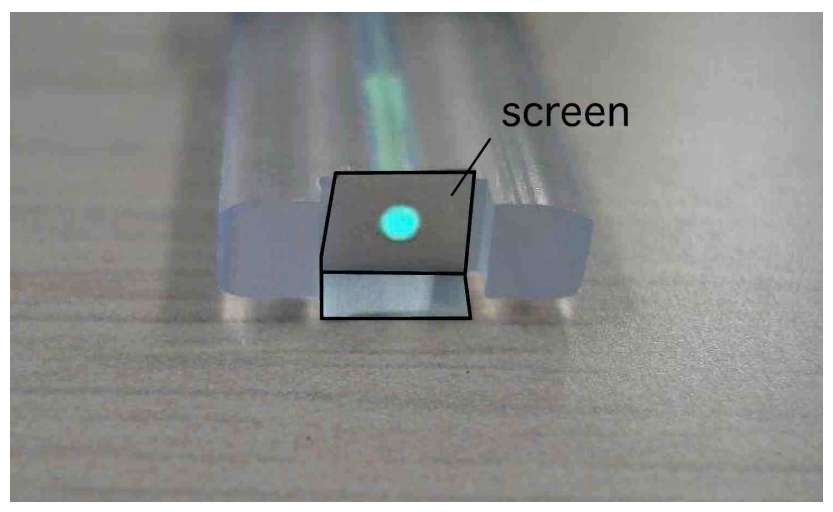

Figure 4: The screen used to block direct scintillation photons. The bright cyan spot is the transverse section of the WLS fibre.
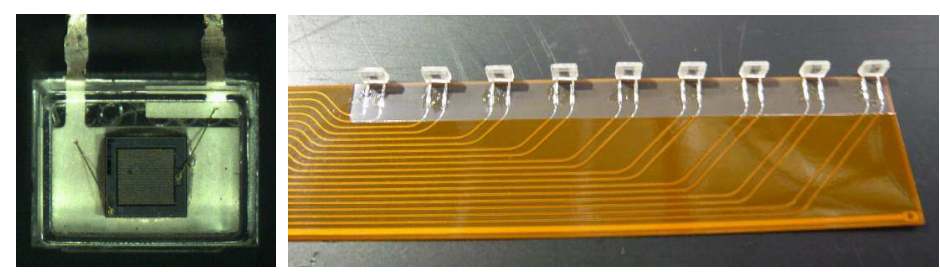

Figure 5: Photographs of a single MPPC (left) and nine MPPCs soldered onto a flat cable (right).

sample of eight, was $14.25 \pm 0.04 \mathrm{~g} / \mathrm{cm}^{3}$, and the mass fractions of elemental components were measured using X-ray diffraction and energy-dispersive X-ray spectroscopy (EDX) to be (tungsten:carbon:cobalt:chrome $)=(0.816: 0.055: 0.125: 0.005)$. The orientation of the scintillator strips in each layer was rotated by $90^{\circ}$ with respect to that of the previous layer.

To monitor the stability of response of each MPPC, a LED-based gain monitoring system was implemented in the prototype. Each of the 18 strips in a given row within a layer was supplied with LED light via a clear optical fibre in which notches had been machined at appropriate positions. Figure 6 shows a photograph of these fibres, in which light can be seen being emitted at the notches. The LED is driven by a dedicated electronic circuit [19. Details of the calibration procedure are discussed in Section 4.1 .

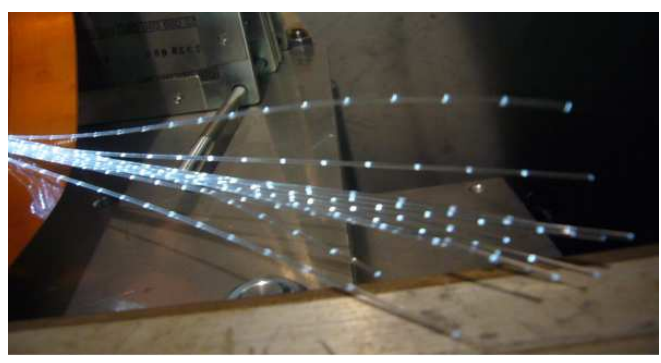

Figure 6: A bundle of clear fibers. Each fibre has 18 notches to supply the LED light to the 18 strips in a row of scintillators. 


\subsection{Data acquisition system}

Nine MPPC signal lines and their power supply lines were grouped together on a flat cable, as noted above, and 12 of these cables were connected to a single base board. The base board contains up to six analogue boards, each of which contained a single ASIC [20,21. Each ASIC controlled 18 MPPCs, such that 108 MPPCs were controlled by one base board. The ASIC performs the following functions:

- fine tuning of MPPC bias voltages via an 8-bit DAC over $4.5 \mathrm{~V}$;

- variable gain charge pre-amplification;

- variable pulse shaping via a $\mathrm{CR}-(\mathrm{RC})^{2}$ shaper, and

- multiplexing 18 channel signals.

This ASIC used a peak-hold method; the hold time was adjusted to give the largest signal when collecting beam data. The ASIC provides both a low- and high-gain operation mode; the low-gain $(8.2 \mathrm{mV} / \mathrm{pC})$ mode was used for the collection of beam runs, while the high-gain $(92 \mathrm{mV} / \mathrm{pC})$ mode was used to collect the LED calibration runs used for MPPC gain monitoring. The optimal hold times were determined separately for both the high-gain and low-gain modes. The spread of gain parameters were $5-10 \%$ 7]. The analogue outputs from the ASICs were sent to VME-based CALICE readout cards (CRC), which provided 16 bit ADCs to digitise the MPPC signals, as well as to perform trigger handling and synchronisation with the data taken by the AHCAL and TCMT prototype.

The raw data from the CRC includes information about the detector configuration, temperature recordings, voltage settings, calibration constants and other specific information associated with the run in addition to the MPPC signals. It was stored in the LCIO format, the standard for ILC R\&D. The actual readout system of the ScECAL prototype was based on that used for the CALICE AHCAL, as described in Ref. [7.

\subsection{Characterisation of MPPCs and their non-linear response}

The prototype used 2160 MPPCs. This subsection discusses how MPPCs were characterised in our laboratory. One of MPPC's characteristics is its non-linear response which is inherent for all SiPMs. The correction for the effects of this non-linear response is discrived in the calibration procedure in Section 4.1 .

MPPC characterisation The gain $G$ of the MPPC is proportional to the excess voltage applied above the breakdown voltage (over-voltage), $\Delta \mathrm{V}$. The gain can therefore be expressed as $G=C \Delta \mathrm{V}$, where $C$ is the average single pixel capacitance of the MPPC. Two sets of MPPCs were used to in the prototype: the first 276 pieces were produced in 2007 while the remaining 1884 were produced in 2008 2. All MPPCs had 1600 pixels in an active area of $1 \times 1 \mathrm{~mm}^{2}$. The properties of all MPPCs in these two sets were measured before constructing the detector prototype. For each MPPC, the gain, noise rate and capacitance were measured as a function of the bias voltage. Figure 7 left shows the distribution of the breakdown voltage of MPPCs, and right shows the extracted single-pixel capacitance $C$ for the two sets. The MPPCs produced in 2008 were used throughout most of the prototype, while those produced in 2007 were installed only in the first and the fourth rows of layers 21-28. By installing the 2007 devices in the layers and rows with low energy deposits, possible systematic effects associated with two sets of sensors are reduced. The $\Delta V$ is tuned to $3.0 \mathrm{~V}$ for all channels in the test beam experiment.

Correction for the non-linear response The non-linear response of MPPCs is approximately described by: ,

$$
F\left(N_{\text {in }}\right):=N_{\text {fired }}=N_{\text {pix }}\left\{1-\exp \left(\frac{-\epsilon N_{\text {in }}}{N_{\text {pix }}}\right)\right\},
$$

\footnotetext{
${ }^{2}$ The bespoke model provided by Hamamatsu K.K. [15] to CALICE was Model MPPC-11-025M, corresponding closely to the commercially available device S10362-11-25P.
} 
where $N_{\text {pix }}$ is the number of pixels on the MPPC, $\epsilon$ is the photon detection efficiency including the normalization factor of PMT, and $N_{\text {in }}$ is the number of incident photons on the MPPC surface.

However, this function requires modification to take into account the possibility that a single pixel may fire more than once during a signal pulse event. The fact that the $12 \mathrm{~ns}$ decay time of a WLS fibre 23] is longer than the 4 ns MPPC pixel recovery time 24, illustrates this phenomenon. In this study the effective number of pixels, $N_{\text {pix }}^{\text {eff }}$, rather than a constant number of pixels, $N_{\text {pix }}$, is used to represent this behaviour. The parameter $N_{\text {pix }}^{\text {eff }}$ was determined empirically through measurements of 72 channels in layer 30 of the prototype by fitting Equation 1 to the signals from these channels. The $30^{\text {th }}$ layer consists entirely of MPPCs produced in 2008. The impact of possible differences in $N_{\text {pix }}^{\text {eff }}$ between the 2007 and 2008 MPPCs is discussed in Section 5.2. The signals are collected using a ps pulsed laser, of wavelength $408 \mathrm{~nm}$ and FWHM $31 \mathrm{p} \$ 3$, after the detector had been disassembled into layers and transported to Matsumoto, Japan. Figure 8 shows a schematic of the setup used to measure the saturating response, while Fig. 9 left shows a typical MPPC response, i.e. the number of MPPC pixels fired as a function of the incident photon signal measured using a PMT. Equation 1, even with $N_{\text {pix }}^{\text {eff }}$, is only applicable within a limited range, outside of which the response function changes at high photon yields, because the recovery of pixels depends on the number of incident photons [25]: a constant parameter, $N_{\text {pix }}^{\text {eff }}$, characterizes the behaviour. The upper limit on the range over which Equation 1 is fitted is based on the point at which the data stop exhibiting exponential behaviour. Figure 9 right shows the gradient of Fig. 9 left with respect to the PMT response. The plot has two distinct regions of approximately linear behaviour on a logarithmic scale. These are fitted separately, and the intersection of these two linear fits is taken as the upper limit of the fit range for Fig. 9 left. To put these effects of non-linear response into context, considering only MPPCs that register a signal during a $32 \mathrm{GeV}$ electron beam run, fewer than $1 \%$ of MPPCs have $N_{\text {fired }}>2000$. Figure 10 shows the distribution of $N_{\text {pix }}^{\text {eff }}$, obtained by fitting the parameters of Equation 1 to measurements from 72 strips in layer 30. This distribution has a mean and a SD of 2428 and 245 pixels, respectively. This mean value was used to implement the correction for the MPPC non-linear response for all channels.

\section{Test beam at FNAL}

\subsection{Beams and setup}

The prototype described in Section 2.1 was exposed to particle beams of varying type and energy at the Meson Test Beam Facility number 6 (MT6) at Fermilab: electrons between 1 and $32 \mathrm{GeV}$ to study the electromagnetic response of the detector; $32 \mathrm{GeV}$ muons for detector calibration; charged pions between 1 and $32 \mathrm{GeV}$ to study the hadronic response in combination with the AHCAL and TCMT. The time structure of the beams was one $4 \mathrm{~s}$ spill per minute in MT6. This paper reports the response of the prototype to the electron beam data collected in May 2009 at energies between $2 \mathrm{GeV}$ and $32 \mathrm{GeV}$.

The setup of the beam line is shown in Fig. 11. A Cerenkov counter 26] placed upstream of the experimental area was used for triggering, together with various combinations of plastic scintillators. A $200 \times 200 \mathrm{~mm}^{2}$ counter provided the trigger signals for muon runs, while a pair of $100 \times 100 \mathrm{~mm}^{2}$ counters provided the trigger signals for pion and electron runs: a coincidence signal from two counters separated by $2.5 \mathrm{~m}$ along the beam direction was required. Additionally, a $1 \times 1 \mathrm{~m}^{2}$ counter with a $200 \times 200 \mathrm{~mm}^{2}$ hole at its centre was used as a veto counter. The combinations of trigger counter and the pressure of the Cerenkov counter nitrogen gas for the electron and muon runs are listed in Table 1. For beam energies $E=8 \mathrm{GeV}$ and $12 \mathrm{GeV}$, two different Črenkov counter pressures were used. The effects of these differences are small and

\footnotetext{
${ }^{3}$ PiL040X (Head) + EIG2000DX (Controller) provided by Advanced Laser Diode System A.L.S. GmbH.
} 

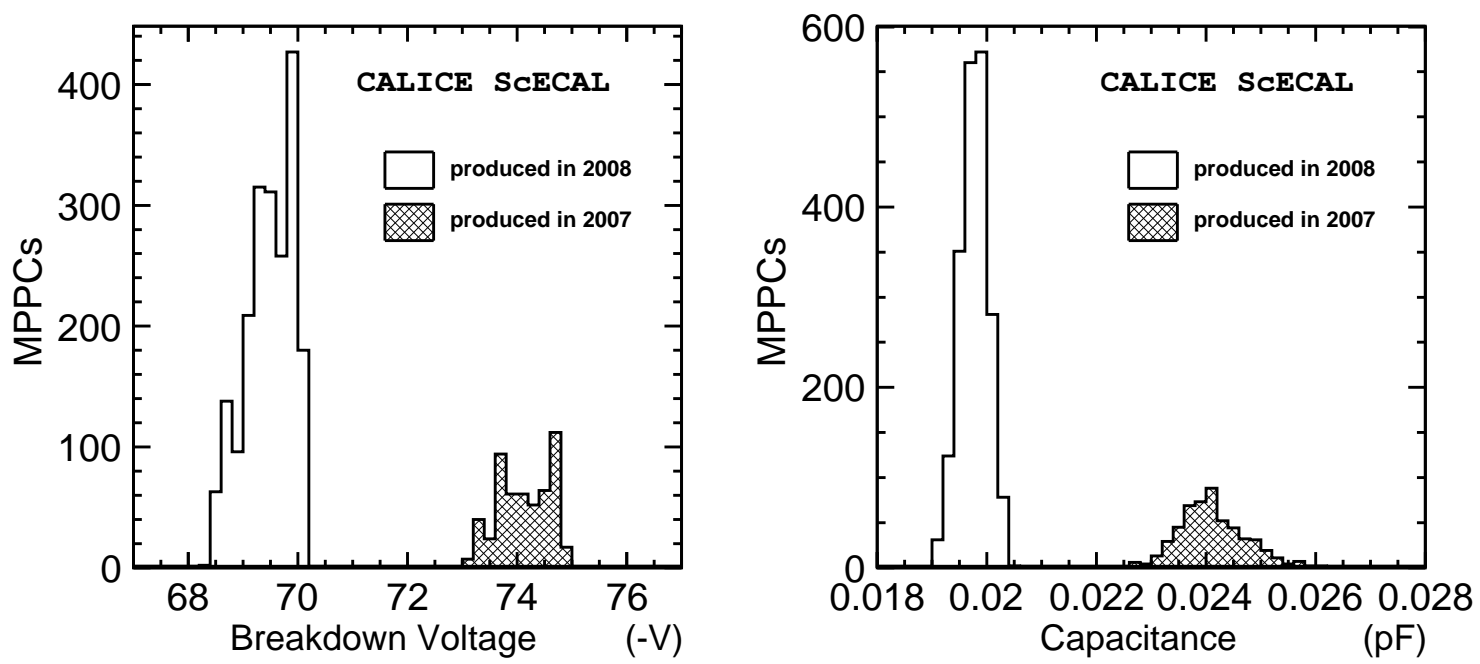

Figure 7: Distributions of breakdown voltage (left) and pixel capacitance (right) of the MPPCs produced in 2007 (hatched) and 2008 (open).

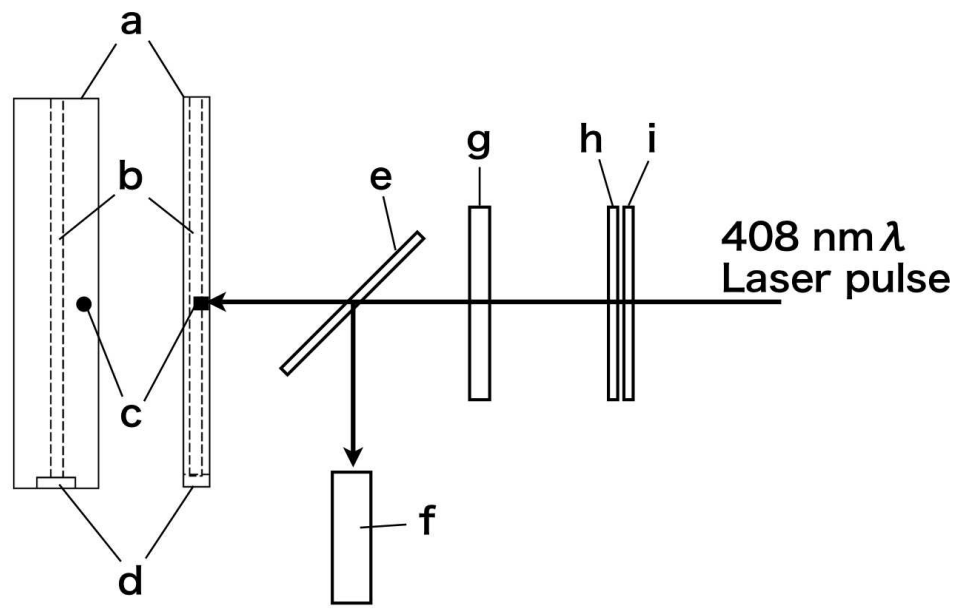

Figure 8: Experimental setup for the $N_{\text {pix }}^{\text {eff }}$ measurement: a) target scintillator wrapped in reflective foil (left top view, right side view); b) WLS fibre; c) irradiation position with a small hole in reflector; d) MPPC; e) semi-transparent mirror; f) photomultiplier tube; g) lens; h) polarising plate (fixed); and i) polarising plate (rotatable). 

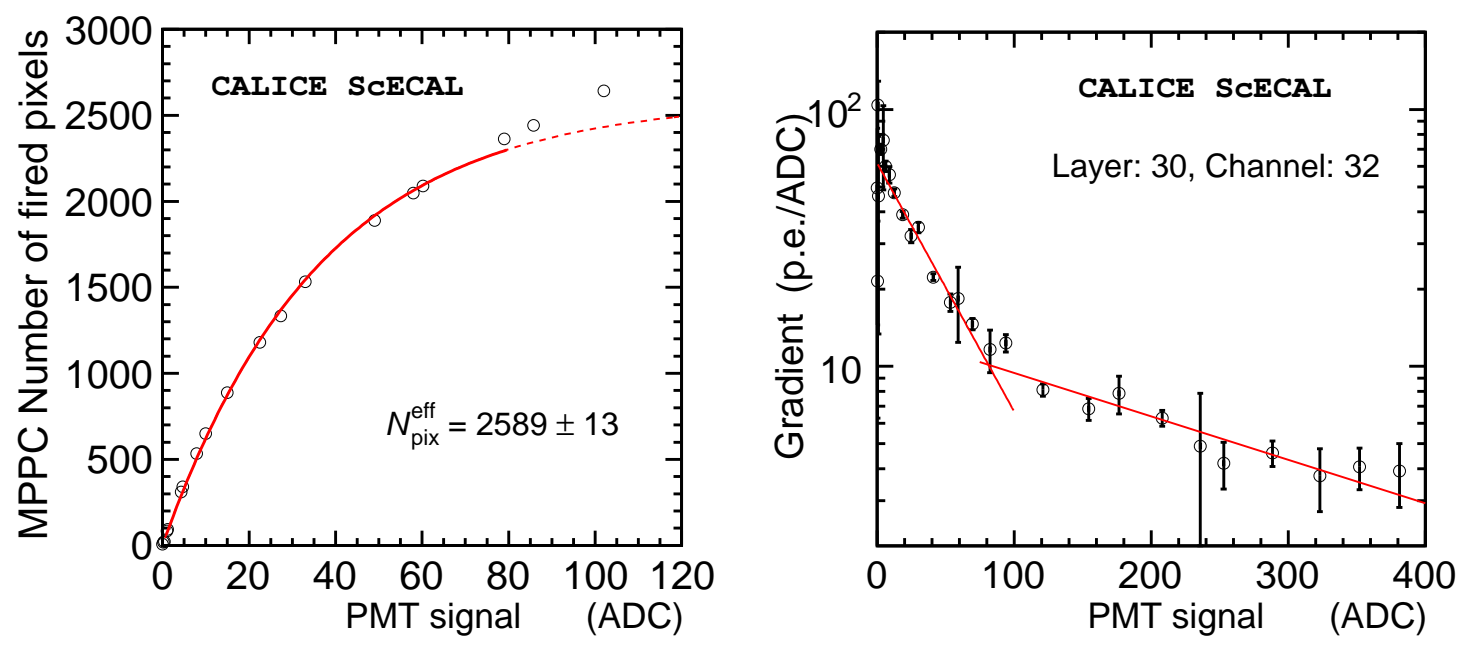

Figure 9: left Number of MPPC pixels fired as a function of the incident photon signal measured using a PMT. The curve shows the results of a fit using Equation 1 the solid line indicates the region over which the fit is performed; the dashed curve shows the extrapolation of the fit results outside this range. right Gradient of left plot vs. PMT signal showing fits in two different regions and their intersection, from which the fitting range of the left plot was determined, see text for details.

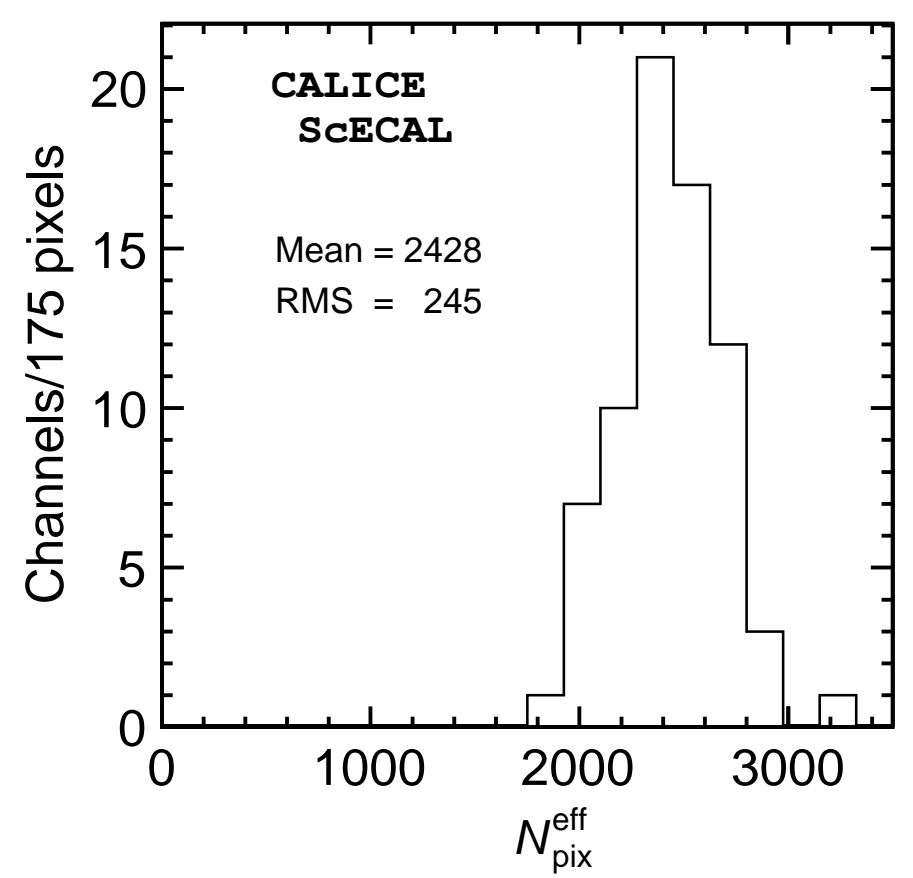

Figure 10: Distribution of the number of effective pixels, $N_{\text {pix }}^{\text {eff }}$, measured in 72 strips. 


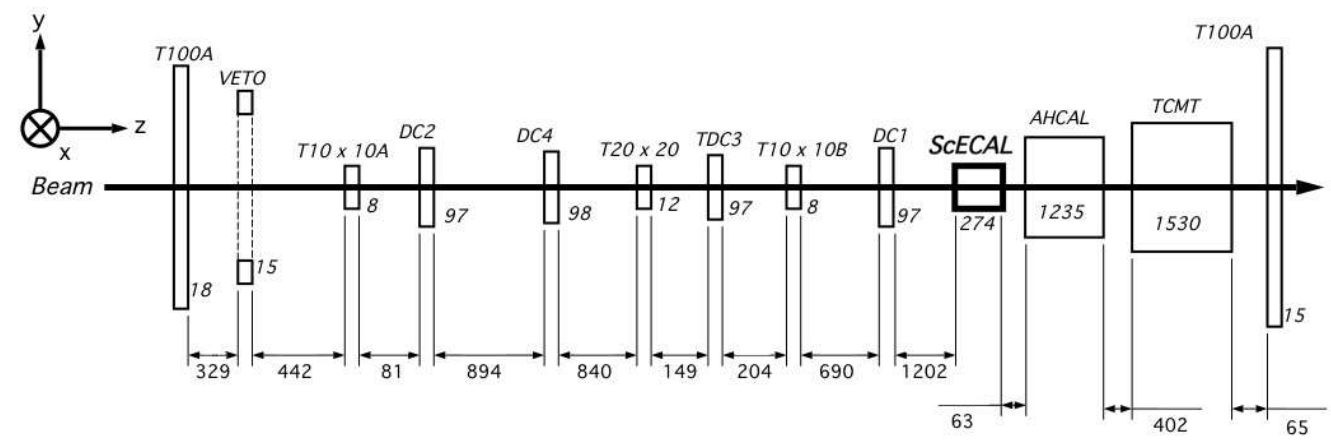

Figure 11: Configuration of detectors on the MT6 beam-line at the MTBF, schematic is not to scale. A right-handed coordinate system is shown. Italic numbers at bottom/right of detector elements show their thicknesses. All dimensions are in mm.

are included in estimating systematic uncertainties. The $200 \times 200 \mathrm{~mm}^{2}$ counter also served as a multiplicity counter to distinguish multi-particle events from single particle events: the signal amplitude of this counter was used to remove multi-particle events in the off-line analysis.

Table 1: Trigger systems used for different particles and energies. The pressure of the Cerenkov counter used for each trigger configuration is also indicated.

\begin{tabular}{lrcc}
\hline \hline Particle & $E[\mathrm{GeV}]$ & Trigger & Čerenkov pressure (hPA) \\
\hline muon & 32 & $200 \times 200 \mathrm{~mm}^{2}$ & - \\
electron & 2 & $100 \times 100 \mathrm{~mm}^{2}$ & 345 \\
electron & 4 & $100 \times 100 \mathrm{~mm}^{2}$ & 345 \\
electron & 8 & $100 \times 100 \mathrm{~mm}^{2}$ & 282,158 \\
electron & 12 & $100 \times 100 \mathrm{~mm}^{2}$ & 158,138 \\
electron & 15 & $100 \times 100 \mathrm{~mm}^{2}$ & 138 \\
electron & 20 & $100 \times 100 \mathrm{~mm}^{2}$ & 138 \\
electron & 30 & $100 \times 100 \mathrm{~mm}^{2}$ & 103 \\
electron & 32 & $100 \times 100 \mathrm{~mm}^{2}$ & 103 \\
\hline
\end{tabular}

\subsection{Temperature measurement}

The temperature of the prototype was measured using two thermocouples, one located on the top of the first ScECAL layer and the other at the bottom of the last layer. Figure 12 shows the temperature of data acquisition periods, averaged over each run with a $1 \mathrm{~Hz}$ data recording rate and over the two sensors. Data were recorded in runs with durations varying between 16 and 85 minutes, and the average temperature of the prototype within a given run was stable to within $0.24^{\circ} \mathrm{C}$. The temperatures recorded varied between $19.0^{\circ} \mathrm{C}$ and $27.5^{\circ} \mathrm{C}$. Although this large variation was caused in part by a malfunction of the air conditioning of the experimental hall over a period of two days at the beginning of the data-taking period, this allowed a robust test of the sensitivity of the ScECAL to be performed and confirms the resilience of the prototype. 


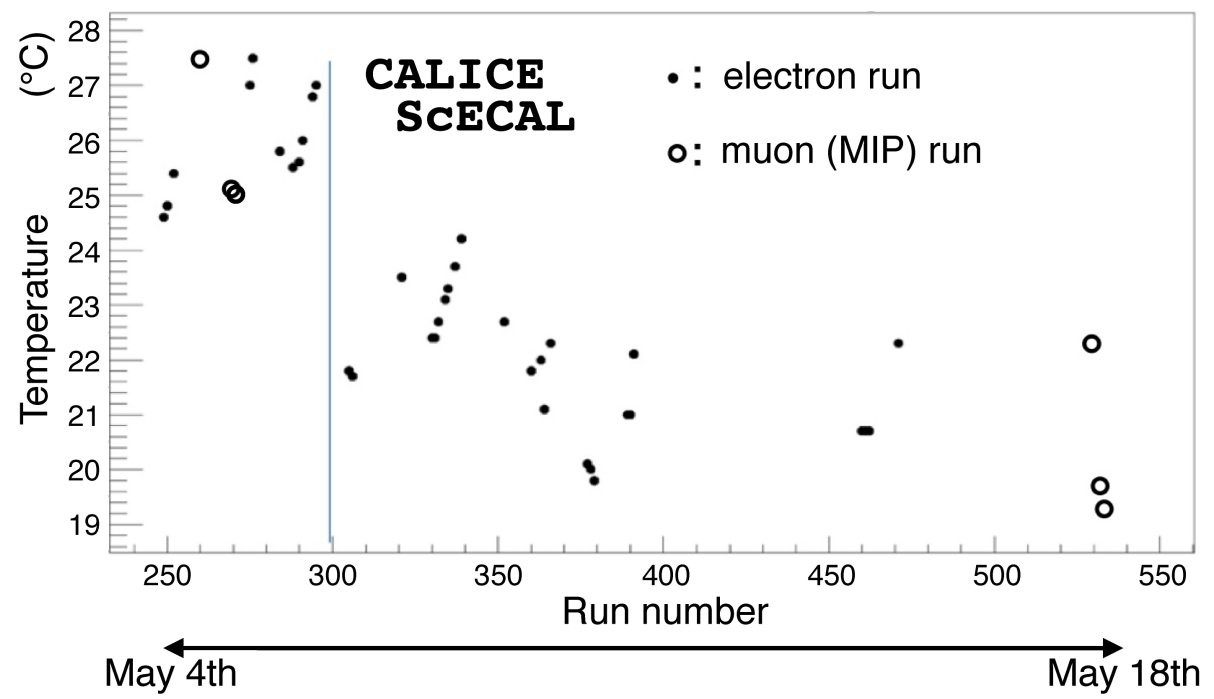

Figure 12: Temperature of the ScECAL prototype during the muon and electron runs collected in 2009. The air conditioning system of the experimental hall was not operational in the period to the left of the vertical line.

\section{Reconstruction procedure}

This section gives an overview of the calibration procedure, the determination of the calibration factors, and the subsequent measurement of the energy spectra.

\subsection{Calibration procedure}

The ScECAL calibration is performed in three steps:

1. relative calibration of cells, to ensure uniform cell-to-cell response;

2. gain calibration (in ADC counts), to determine the signal amplitude corresponding to a single fired pixel, and

3. calibration to an absolute energy scale, using electromagnetic showers.

For the first step, the cell-to-cell response of cells is normalised using the response of each cell to beams of muons, which approximate minimum ionising particles (MIP). The most probable value (MPV) of the signal distribution obtained using muons and measured in ADC counts, $c^{\mathrm{MIP}}$, is the calibration factor of this procedure. After this calibration, the visible energy in the detector is expressed in units of MIPs.

A second calibration step is performed to correct for the non-linear response of MPPCs. The inverse of Equation 1 unfolds the effects of non-linear response as discussed in Section 2.3. However, as $F^{-1}$ is a function of the number of fired pixels, the amplitude of the signal must be converted accordingly. The relevant ADC-photoelectron conversion factor ( $\left.c^{\text {p.e. }}\right)$ is determined in situ for each channel, where a photoelectron corresponds an electron-hole pair triggering a geiger discharge of a pixel. This is an essential role for the LED-based gain monitoring system discussed in Section 2.1.

The second step - the calibration of each MPPC - includes one additional calibration coefficient, because $c^{\text {p.e. }}$ is measured using a high-gain amplifier to achieve a sufficient separation of photoelectron peaks [7, whereas the signals in physics data taking are acquired using a lower gain due to the wider dynamic range required. This calibration coefficient, referred to as intercalibration coefficient $\left(c^{\text {inter }}\right)$, is measured for each channel as the ratio of the amplitudes of the response to LED light with the high-gain to the low-gain settings. Therefore, a $c^{\text {inter }}$ includes 
not only the ratio of amplifier in electronics but also the effect from difference of pulse shape time between the high-gain and low-gain modes.

These calibration constants, namely the ADC-MIP conversion factor, the ADC-photoelectron conversion factor and the inter-calibration coefficient for each channel, were determined in situ and are discussed in Section 4.3. With these constants, a signal in channel $i$ for the physics study can be written as:

$$
A_{i}^{\text {corr }}[\mathrm{MIP}]=F^{-1}\left(A_{i}[\operatorname{ADC}](T) \frac{c_{i}^{\text {inter }}}{c_{i}^{\text {p.e. }}(T)}\right) \frac{c_{i}^{\text {p.e. }}(T)}{c_{i}^{\text {inter }} \cdot c_{i}^{\mathrm{MIP}}(T)},
$$

where $A_{i}[\mathrm{ADC}](T)$ is the uncorrected signal of the cell in ADC counts for a detector of temperature $T$, and $F^{-1}$ has a parameter $N_{\text {pix }}^{\text {eff }}$ instead of $N_{\text {pix }}$ as discussed in Section 2.3, and $\epsilon$ is cancelled in Equation 2 because both a $F^{-1}$ and a $c^{\mathrm{MIP}}$ are inversely proportional to the $\epsilon$.

Each calibration factor is determined as a function of temperature. The sum of these signals represents the energy of an event in a physics run, in units of MIPs, and is given by

$$
E_{\text {reco }}[\mathrm{MIP}]=\sum_{\text {all strips }} A_{i}^{\text {corr }}[\mathrm{MIP}]
$$

The mean of $E_{\text {reco }}$ as a function of the incident beam energy represents the calibration of the ScECAL to an energy scale in $\mathrm{GeV}$ as required for the third calibration step. The demonstration of this calibration is one of the primary goals of the test beam activity reported in this article. Detailed results are discussed in Section 5 .

\subsection{Calibration runs and pedestal measurements}

To determine the $c^{\mathrm{MIP}}$, six muon runs were recorded over a wide range of temperatures, allowing the temperature dependence to be quantified. To determine the $c^{\text {p.e. }}$, several LED calibration runs were typically recorded per day. During each run of 50000 events, the LED power was changed in eleven steps to ensure that some events with a suitable photon yield were present in all channels.

Inter-calibration runs were also taken in the LED calibration runs. For these, intermediate intensity LED light was injected into each channel and the signal in ADC counts was measured in both the low-gain and high-gain modes. In each such run, 50000 events were taken for each of the eleven different LED power settings.

The signal pedestals were monitored by recording 500 randomly triggered events in the period between beam spills. The mean values of these pedestal events were calculated separately for each channel, and subtracted from signals collected during the subsequent set of beam events. The widths of the pedestals were also calculated as the RMS for each channel.

\subsection{Determination of calibration constants}

Three calibration factors, $c^{\mathrm{MIP}}, c^{\text {p.e. }}$ and $c^{\text {inter }}$, discussed in Section 4.1 are determined in this subsection. The methods through which these are also evaluated are described.

\subsubsection{ADC-MIP conversion factor}

To select muon events hits were required to be present in at least ten layers in the same lateral strip position of the same oriented layers of $y$-having detail segmentation in $x$ - (x layer) or $x$-having detail segmentation in $y$ - ( $y$ layer $)$. A strip was defined to have been hit if the recorded signal value was more than three times the width of a Gaussian function fitted to the corresponding pedestal distribution. As an example, Fig. 13 shows the distribution of signal recorded in a typical single strip for muon events, fitted with a Gaussian-convoluted Landau function. The MPV of the function is taken to be the $c^{\mathrm{MIP}}$, and the mean uncertainty of all channels on the fitted MPV was $(1.8 \pm 0.7) \%$. 


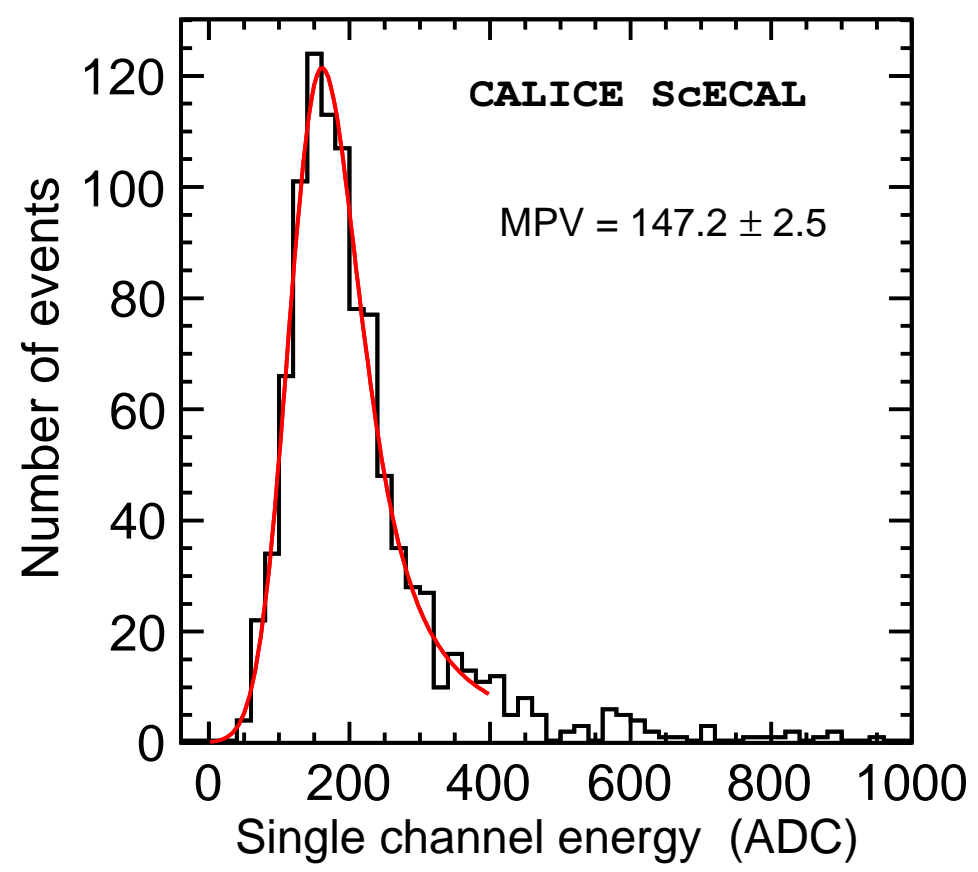

Figure 13: Distribution of pedestal-subtracted energy deposits from MIP-like particles in a single (typical) channel. The solid line shows the result of a fit using a Gaussian-convoluted Landau function.

The MPV of each channel was measured in six dedicated runs at various temperatures, allowing the temperature dependence of the MIP response to be determined. This is illustrated for a typical channel in Fig. 14, showing a linear dependence of the Landau MPV on the average temperature during a run. The $c^{\mathrm{MIP}}$ is therefore expressed as

$$
c^{\mathrm{MIP}}(T)=c^{\mathrm{MIP}}\left(T_{0}\right)+\frac{\mathrm{d} c^{\mathrm{MIP}}}{\mathrm{d} T}\left(T-T_{0}\right),
$$

where $T$ is the temperature at which the measurement was taken, and $T_{0}$ is a reference temperature. The parameters $c^{\mathrm{MIP}}\left(T_{0}\right)$ and $\mathrm{d} c^{\mathrm{MIP}} / \mathrm{d} T$ were determined for each channel and account for the effect of temperature on the energy deposit as measured in each channel. Figure 15 shows the distributions of the $c^{\mathrm{MIP}}$, estimated at $20^{\circ} \mathrm{C}$, and $\left(\mathrm{d} c^{\mathrm{MIP}} / \mathrm{d} T\right) / c^{\mathrm{MIP}}$.

\subsubsection{ADC-photoelectron conversion factor}

The $c^{\text {p.e. }}$ was determined by measuring signal distributions consisting of a few peaks of photoelectrons induced by LED light during the dedicated runs discussed in Section 4.2 Figure 16 shows an example of an MPPC signal distribution, for one of several LED intensities used during a calibration run. The pedestal and first two peaks of photoelectrons are easily distinguished. Three Gaussian functions are used to fit this distribution, with six free parameters: the amplitudes of the three Gaussian functions; the peak position of the first Gaussian function; a sigma equal for all three functions, and an equal distance between adjacent peaks, corresponding to the MPPC gain. The latter parameter is the $c^{\text {p.e. }}$ of this channel. Where successful fits were obtained for more than one of the LED intensities used during the calibration runs, a weighted average of $c^{\text {p.e. }}$ was used. The mean fractional uncertainty on $c^{\text {p.e. }}$, averaged over all channels and temperatures, is $(0.7 \pm 0.3) \%$. 


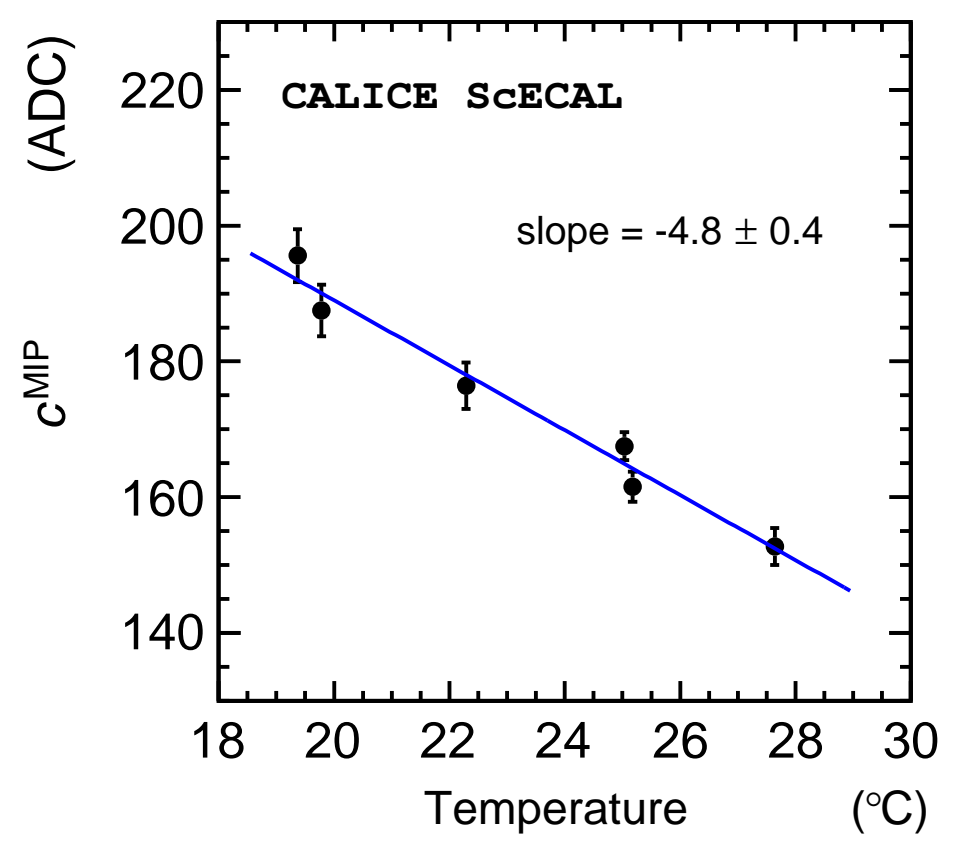

Figure 14: Measured $c^{\mathrm{MIP}}$ of a typical channel as a function of the average detector temperature during a data taking run. The line shows the result of a linear fit. This fitting gives $c^{\mathrm{MIP}}\left(20^{\circ} \mathrm{C}\right)=189 \pm 14$. This channel used a MPPC from the 2008 production.
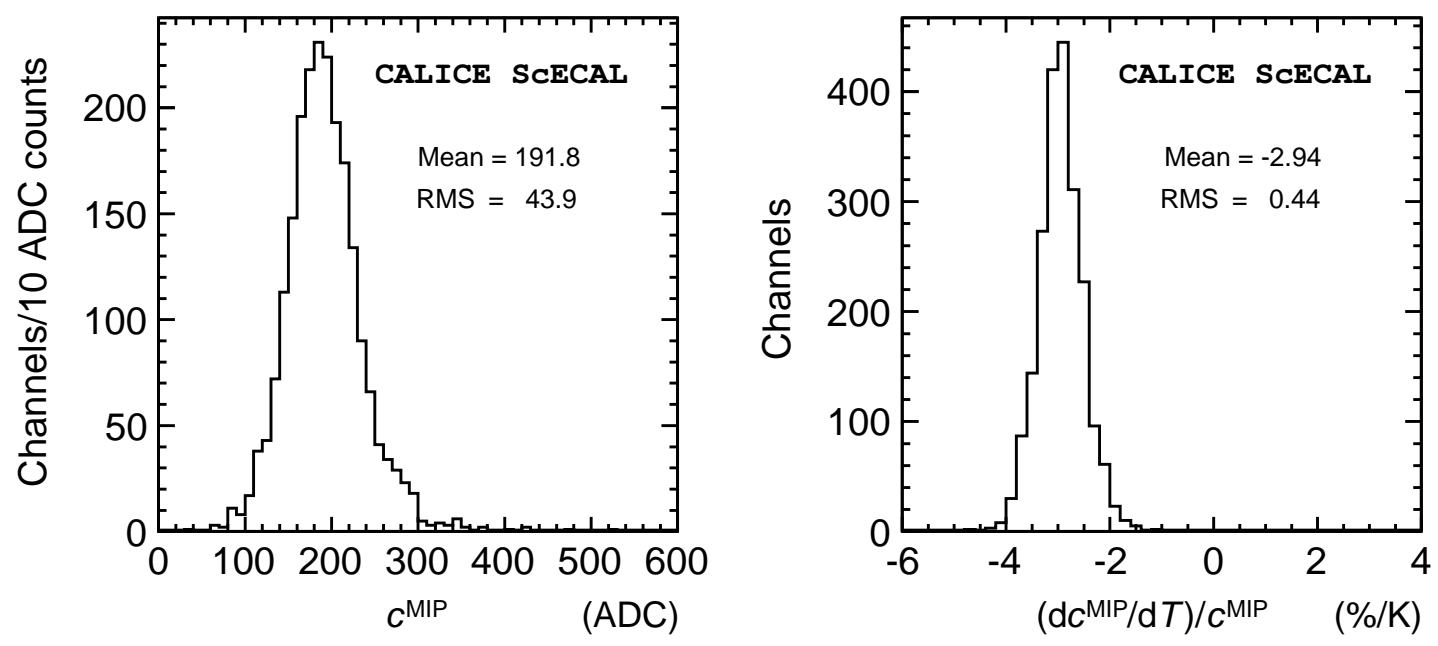

Figure 15: left Distribution of $c^{\mathrm{MIP}}\left(T_{0}=20^{\circ} \mathrm{C}\right)$. right Distribution of the slope of $c^{\mathrm{MIP}},\left(\mathrm{d} c^{\mathrm{MIP}} / \mathrm{d} T\right) / c^{\mathrm{MIP}}$. 


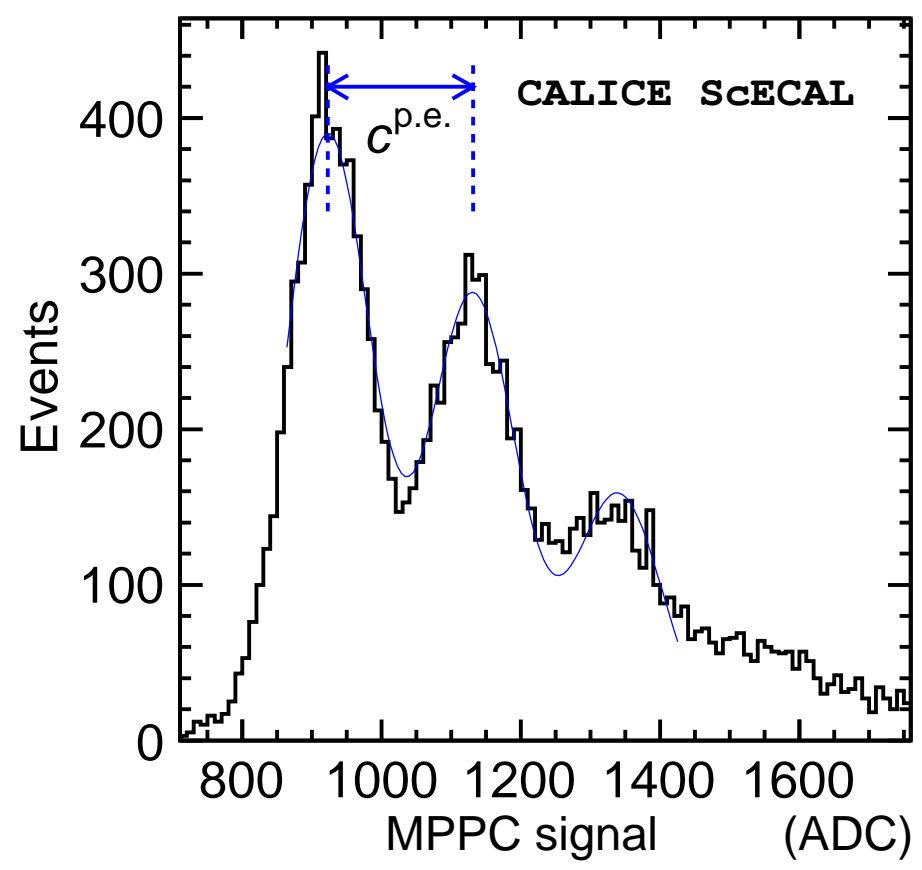

Figure 16: Typical spectrum of a LED run for a single channel, with the results of a three-Gaussian function fit overlaid. The arrow indicates $c^{\text {p.e. }}$ for this channel.

The LED data were collected in nine runs and the variation in conditions between these runs allowed the temperature dependence of $c^{\text {p.e. }}$ to be determined. The $c^{\text {p.e. }}$ was parametrised in the same way as $c^{\mathrm{MIP}}$, assuming a linear dependence with temperature. Approximately $80 \%$ of all channels were calibrated using the LED system. In the remaining channels, either the pedestals had two peaks because of noise in the LED circuit or the peaks of photoelectrons were not sufficiently distinct as to be separable. The majority of these were concentrated within a few rows of channels, mostly located on the first or fourth (outermost) rows of layers. The two-peak pedestals were not observed during physics runs. Figure 17 shows the distributions of $c^{\text {p.e. }}\left(\right.$ at $\left.20^{\circ} \mathrm{C}\right)$ and $\left(\mathrm{d} c^{\text {p.e. }} / c^{\text {p.e. }}\right) / \mathrm{d} T$ for completeness. The temperature dependence of $c^{\text {p.e. }}$ is only affected by gain variations, whereas the dependence of $c^{\mathrm{MIP}}$ on temperature includes contributions from both variations in gain and variations in the photon detection efficiency.

Where they were available, these $c^{\text {p.e. }}(T)$ values were used to apply the correction of MPPC non-linear response to electron data at temperature $T$, channel-by-channel. The following criteria were also required: $170<c^{\text {p.e. }}\left(T_{0}=20^{\circ} \mathrm{C}\right)<260 \mathrm{ADC}$ counts/photoelectron; the corresponding fit uncertainty between 0.2 and $50 \mathrm{ADC}$ counts/photoelectron. For channels where successful fits were not obtained, the average value of successfully fitted channels was used: $77 \%$ of all channels have individual $c^{\text {p.e. }}\left(T_{0}=20^{\circ} \mathrm{C}\right)$. A single value for $\left(\mathrm{d} c^{\text {p.e. }} / \mathrm{d} T\right) / c^{\text {p.e. }}$, taken from the mean of the Gaussian fit as shown in Fig. 17, is used for all channels.

\subsubsection{Inter-calibration constant}

The dedicated inter-calibration runs used LED light of higher intensity, which could be measured in both high-gain and low-gain modes of the ASIC. Figure 18 left compares MPPC response in the two operating modes for the same LED power. A $c^{\text {inter }}$ for each channel was determined as

$$
c^{\text {inter }}=\frac{\left\langle A_{i}^{\text {high }}\right\rangle}{\left\langle A_{i}^{\text {low }}\right\rangle},
$$



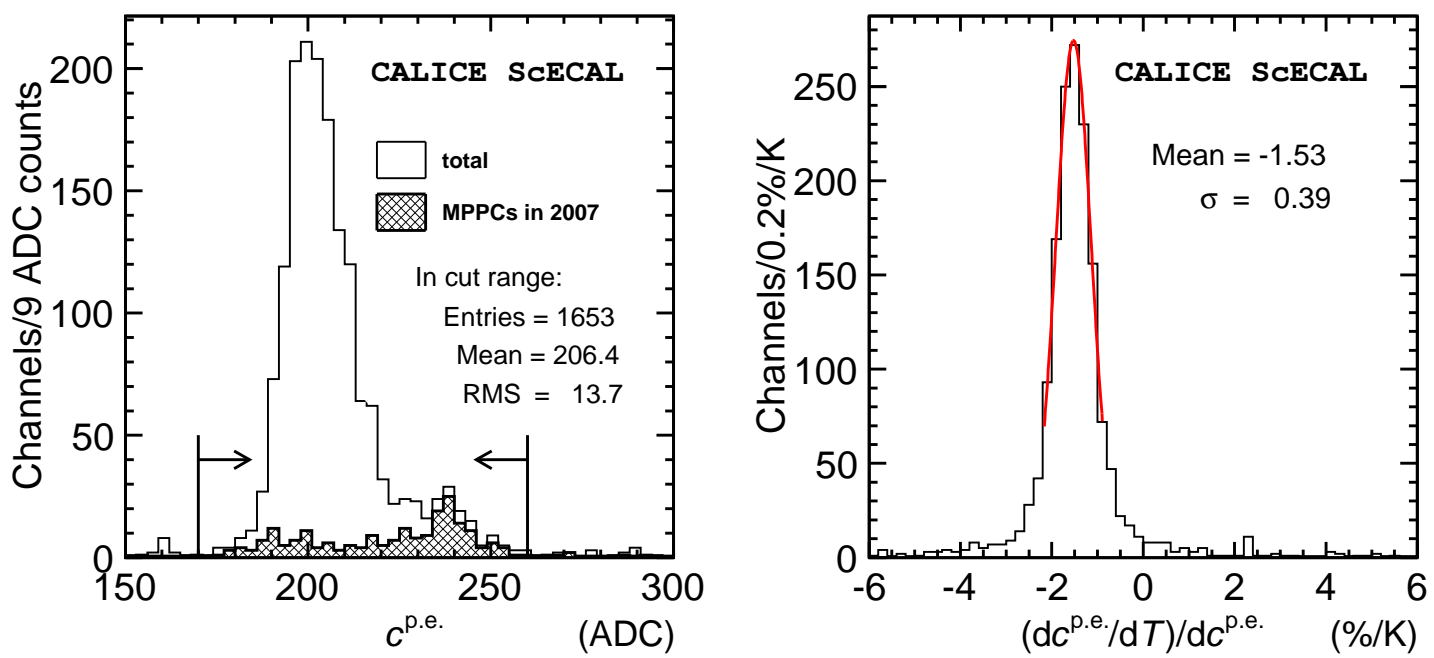

Figure 17: left Distribution of $c^{\text {p.e. }}\left(T_{0}=20^{\circ} \mathrm{C}\right)$. MPPCs produced in 2007 and 2008 have different characteristics (cf. Fig. 77). right Distribution of $\left(\mathrm{d} c^{\text {p.e. }} / \mathrm{d} T\right) / c^{\text {p.e. }}$. The curve shows the result of a Gaussian fit used to extract the mean and SD.

where $A_{i}^{\text {high }}$ and $A_{i}^{\text {low }}$ are the signal amplitudes in the high-gain and low-gain modes of preamplifiers, respectively.

As shown in Fig. 18 right, the distribution of $c^{\text {inter }}$ has a tail extending to large values. These channels have unexpectedly small $A_{i}^{\text {high }}$ and $A_{i}^{\text {low }}$ values due to insufficient light supplied by the LED even at its highest power setting. Most of these channels were located at the far end of the fibres distributing the LED light. An additional contribution to large $c^{\text {inter }}$ values in such cases is a possible downward pedestal shift during LED runs due to large power consumption of other highly illuminated channels. The impact of this effect should be more pronounced for small values of $A_{i}^{\text {low }}$. Having confirmed d that the large $c^{\text {inter }}$ does not represent real $c^{\text {inter }}$, we replaced the $c^{\text {inter }}$ value of all channels that are more than 2 RMS above the mean of the entire distribution by the mean calculated using only channels that are below this boundary. The sensitivity of the energy resolution to the choice of this boundary is taken into account as a potential systematic uncertainty.

\subsection{Reconstruction of electron energy spectra}

The energy $E$ of events recorded during electron runs were reconstructed according to Equations 2 and 3 as discussed in Section 4.1. In this reconstruction procedure, signals that are less than three RMS above the mean were rejected in both data and the simulation. Events recorded in electron runs that are triggered by the Cerenkov counter still include contamination from pions, muons, and multi-particle events. To further enhance the purity of the electron sample and to select events that are contained within the fiducial volume of the ScECAL prototype, the following selection criteria were applied:

1. the layer with maximum reconstructed energy must be within the first 20 ScECAL layers;

2. the reconstructed energy in this layer must exceed a beam energy dependent threshold, as given in Table 2,

3. the reconstructed energy of the highest energy AHCAL layer must be less than 20 MIPs;

\footnotetext{
${ }^{\mathrm{d}}$ Exchanging the electronics of the DAQ for those channels with normal channels confirmed that the large $c^{\text {inter }}$ was not due to a genuine change of $A_{i}^{\text {high }} / A_{i}^{\text {low }}$. This behavior on those channels was uncorrelated with $c^{\text {p.e. }}$. The $c^{\mathrm{MIP}}$ on those channels shows no correlation with $c^{\text {inter }}$.
} 

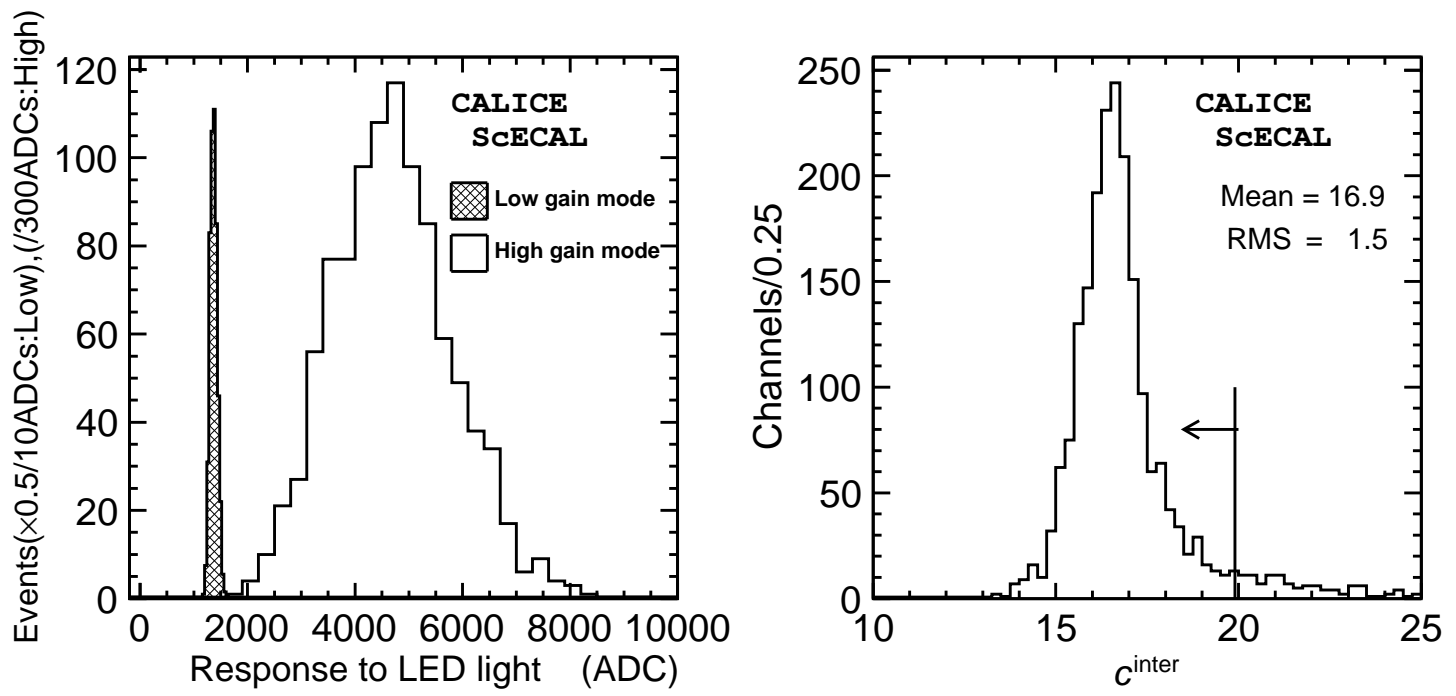

Figure 18: left: Distributions of the MPPC response to LED light in an inter-calibration run before pedestal subtraction, using the high-gain mode (open) and the low-gain mode (hatched). The same LED power was supplied in both cases. right: Distribution of the inter-calibration constant $c^{\text {inter }}$. The arrow shows the boundary above which an average value of $c^{\text {inter }}$ is used, to avoid excessively large $c^{\text {inter }}$ values, see text for details.

4. the reconstructed energy in the most downstream layer of the AHCAL must be less than 0.4 MIPs;

5. the energy-weighted mean position - measured using only $x$ layers - of ScECAL hits must be within $40 \mathrm{~mm}$ of the detector centre in the $x$ direction;

6. equivalent of criterion 5 in $y$; and

7. the multiplicity counter signal should correspond to less than 1.4 MIP [26],

where the first three criteria reduce contamination from both pions and muons, the fourth further reduces that of muons, the fifth and sixth define the lateral fiducial area and the seventh reduces the selection of multi-particle events.

Table 2: Energy thresholds required for the layer with maximum reconstructed energy.

\begin{tabular}{cc}
\hline \hline $\begin{array}{c}\text { Beam energy } \\
{[\mathrm{GeV}]}\end{array}$ & $\begin{array}{c}\text { Minimum energy } \\
\text { reconstructed [MIP] }\end{array}$ \\
\hline 2 & 15 \\
4 & 27 \\
8 & 54 \\
12 & 80 \\
15 & 95 \\
20 & 125 \\
30 & 200 \\
32 & 200 \\
\hline
\end{tabular}

Figure 19 shows the reconstructed energy spectrum of events recorded in a single $2 \mathrm{GeV}$ electron run and a single $32 \mathrm{GeV}$ electron run after the sequential application of these selection 

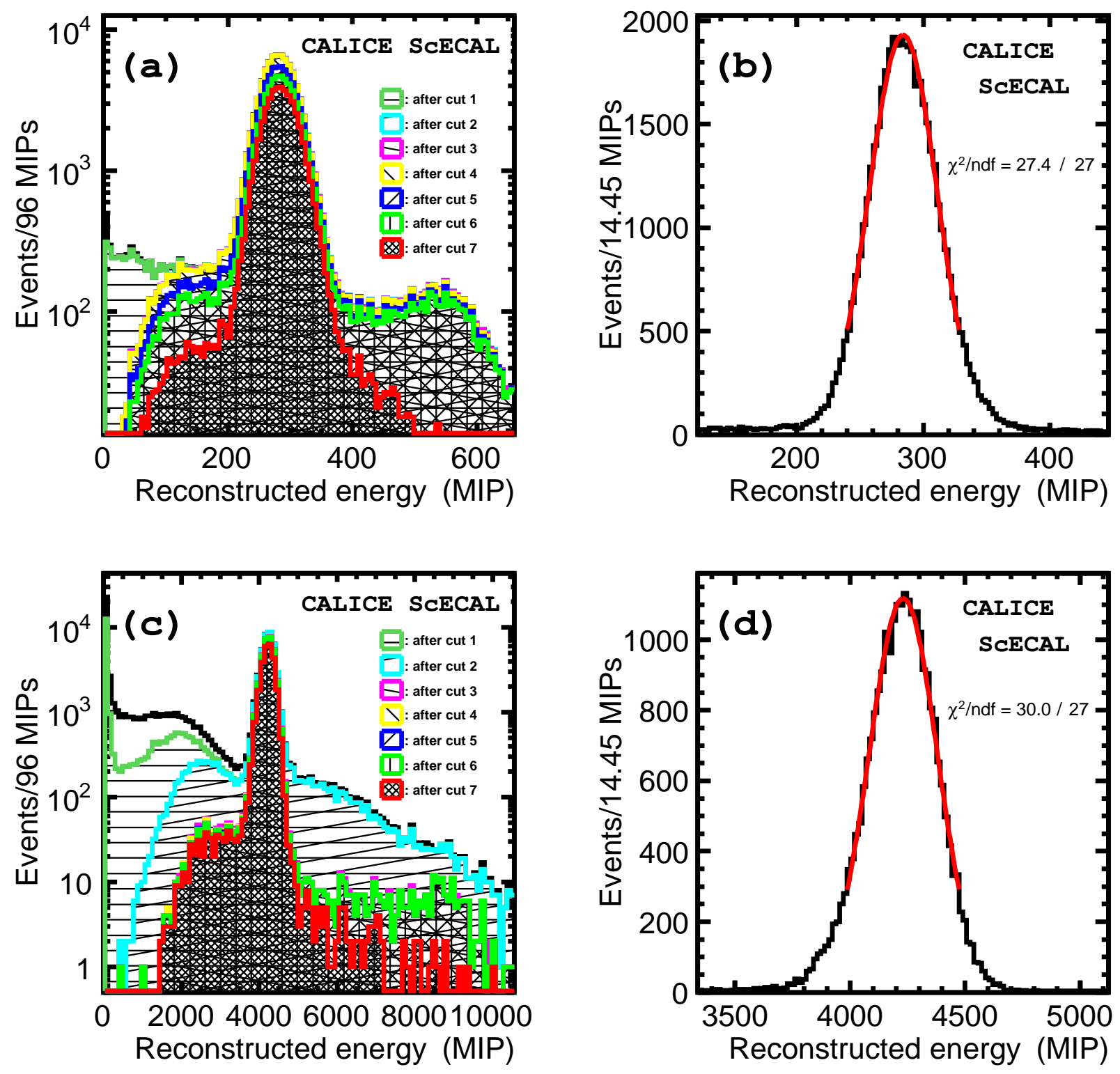

Figure 19: Energy spectra of events collected in a $2 \mathrm{GeV}$ electron run (top: a, b), and $32 \mathrm{GeV}$ electron run (bottom: c, d). Left: (a, c) show the effects of the sequential application of selection criteria 1-7, see text for details. Right: (b, d) show the spectrum after all cuts. A solid curve in (b) and (d) is the result of a Gaussian fit in a range that contains $90 \%$ of selected events. 
cuts, and the energy spectrum after all cuts. These criteria remove almost all two-particle events, which are identified as having higher energies than the main electron peak. The residual contamination form particles other than electrons, associated with energies below the main peak, is less than between $0.1 \%(32 \mathrm{GeV})$ and $1 \%(2 \mathrm{GeV})$. The final spectrum is described well by a Gaussian function in a range of $\pm 1.65 \sigma$. The reduced $\chi^{2}$ of the fits to the spectra collected at all energies were between 0.9 and 1.2. The mean reconstructed energy, $E_{\mathrm{reco}}$, and resolution, $\sigma_{E}$, were obtained from the mean and width of the Gaussian function fitted to the reconstructed energy spectra. The relative resolution is calculated as a ratio $\sigma_{E} / E_{\text {reco }}$. Data from runs with the same nominal beam energy were combined, weighted by their statistical uncertainties.

\section{Performance of the prototype}

\subsection{Mean and resolution with statistical uncertainties}

Table 3 summarises the mean energy response and resolution for each beam energy, together with their statistical uncertainties. Figure 20 shows the energy resolutions of the five runs collected at $4 \mathrm{GeV}$. The variations measured in different runs at the same nominal energy are consistent within their statistical uncertainties, as are those at other beam energies.

Table 3: Mean reconstructed energy and relative resolution for the combined data sets. The resolution includes the intrinsic energy spread of the beam. The uncertainties are statistical only.

\begin{tabular}{crc}
\hline \hline Beam energy $[\mathrm{GeV}]$ & \multicolumn{1}{c}{$E_{\text {reco }}[\mathrm{MIP}]$} & $\sigma_{E} / E_{\text {reco }}(\%)$ \\
\hline 2 & $281.53 \pm 0.08$ & $9.633 \pm 0.035$ \\
4 & $545.10 \pm 0.12$ & $6.855 \pm 0.026$ \\
8 & $1076.52 \pm 0.14$ & $5.049 \pm 0.015$ \\
12 & $1588.43 \pm 0.22$ & $4.388 \pm 0.016$ \\
15 & $1966.31 \pm 0.23$ & $4.222 \pm 0.014$ \\
20 & $2589.30 \pm 0.29$ & $3.791 \pm 0.013$ \\
30 & $3910.4 \pm 0.6$ & $3.445 \pm 0.017$ \\
32 & $4201.5 \pm 0.7$ & $3.425 \pm 0.020$ \\
\hline
\end{tabular}

In contrast, the mean reconstructed energies measured in other runs show variations that are beyond what is expected from their statistical uncertainties, as seen in Fig. 21. Imperfections in the correction for temperature variation were considered as a possible explanation for this difference. However, Fig. 21 shows that the correlation between the reconstructed energy of individual runs and the temperature is only apparent for runs taken at $8 \mathrm{GeV}, 12 \mathrm{GeV}$, and $20 \mathrm{GeV}$. The following subsections discuss investigations into potential sources of systematic effects that may account for these differences.

\section{$5.2 \quad$ Systematic uncertainties}

We consider sources of potential systematic uncertainty from the event selection criteria, the calibration factors/constants, correction of the MPPC non-linear response and the beam energy spread. Table 4 lists the contributions from different sources to the overall uncertainty for each beam energy.

\section{Event selection}

As discussed in Section 4.4, seven cuts were used to select well-contained electron events. To estimate the systematic uncertainties associated with these selection criteria, the impact of varying the applied cuts was evaluated. The largest contribution to the energy mean 


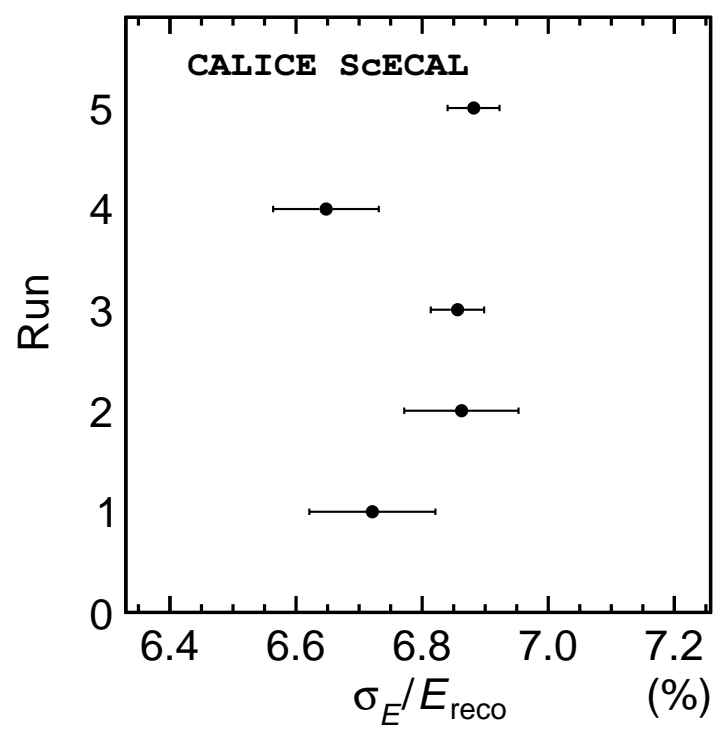

Figure 20: The energy resolution obtained from the five electron runs collected at a beam energy of $4 \mathrm{GeV}$.

arises from the fiducial volume cut in the $x$ direction, due to the larger beam spread in $x$ than in $y$.

\section{ADC-MIP conversion factor}

Systematic uncertainties on the ScECAL performance originating from the statistical uncertainty in the extraction of $c^{\mathrm{MIP}}$ were estimated. The assumed values of $c^{\mathrm{MIP}}\left(T_{0}\right)$ and $\mathrm{d} c^{\mathrm{MIP}} / \mathrm{d} T$ were randomly fluctuated around their central values using a Gaussian probability distribution function (PDF) with a width equal to the statistical uncertainty of these parameters extracted for each channel. The data were re-analysed twenty times using such fluctuated calibrations. The absolute value of the systematic uncertainty from $c^{\mathrm{MIP}}\left(T_{0}\right)\left(\mathrm{d} c^{\mathrm{MIP}} / \mathrm{d} T\right)$ on the reconstructed energy mean is less than $0.3 \%(0.06 \%)$ for all beam energies.

\section{ADC-photoelectron conversion factor}

A similar method is applied to systematic uncertainties originating from $c^{\text {p.e. }}$, and effects on the reconstructed mean energies were found to be negligible.

\section{Inter-calibration constant}

Systematic effects arising from uncertainties in the inter-calibration constants are also studied using a pseudo-experiment method. In the case of channels with a successfully measured inter-calibration constant, the constant is varied according to a Gaussian PDF, whose width is the uncertainty of the inter-calibration constant of the channel under consideration. In the case of channels where the measurement was not successful, the SD of inter-calibration constants for all measured channels was used as the width of the Gaussian PDF. On the basis of twenty such pseudo-experiments, changes in performance were negligibly small. As discussed in Section 4.3.3. the value of $c^{\text {inter }}$ in any channel that was more than $2 \sigma$ above the mean of all channels, was replaced by the mean value itself. To investigate the effect of this procedure, the criterion of the $c^{\text {inter }}$ cut was changed from $1 \sigma$ to $3 \sigma$ and also for the case of all measured $c^{\text {inter }}$. The relative shifts found in the mean and the resolution of energy with respect to the default case were less than $0.01 \%$ when changing the criterion from 1 to $3 \sigma$ for all energies, and less than $0.1 \%$ when all 


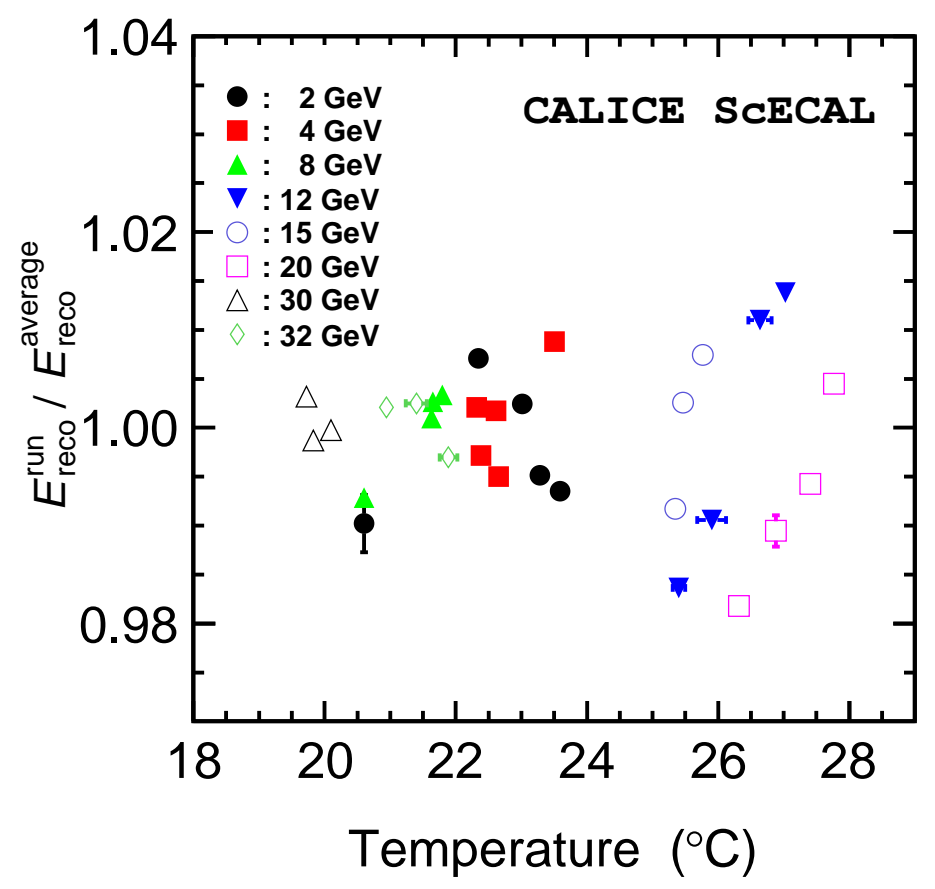

Figure 21: Ratio of the reconstructed mean of individual runs to the average of all runs at a given beam energy including systematic uncertainties $v s$. temperature during data taking.

measured $c^{\text {inter }}$ were used. Therefore, the systematic uncertainties from this procedure are also considered to be negligible.

The number of effective pixels of the MPPC

The number of effective MPPC pixels, $N_{\text {pix }}^{\text {eff }}$, was measured in 72 strips. The mean of these 72 measurements was used when applying correction of the MPPC non-linear response to all strips of the prototype. Pseudo-experiments in which $N_{\text {pix }}^{\text {eff }}$ of each strip was varied with a Gaussian PDF were performed to study the impact of the uncertainties of this quantity. The width of the Gaussian PDF was taken as the SD of the 72 measurements. Effects on calorimeter performance were rather small: the absolute value of the systematic uncertainty from the uncertainty of the number of effective pixels is less than $0.13 \%$ for all beam energies. The $72 \mathrm{MPPC}$ samples are all from the 2008 production. A $N_{\text {pix }}^{\mathrm{eff}}$ of the 2007 production is estimated to be 2185 , which is within one SD of of the 2008 products. We estimated this value using data from the first prototype where all MPPCs were 2007 products [11. Additionally, the 2007 products only represent $13 \%$ of all MPPCs in the prototype and these are all located in peripheral regions. Therefore, we ignore the effect of differences between the 2008 and 2007 devices.

\section{Response dependence on hit position along the strip length}

A previous ScECAL prototype using extruded scintillator strips demonstrated a significant dependence of the response on the hit position along the scintillator strips [27. This response non-uniformity results in a significant degradation of the energy resolution.

Applying a screen in front of the MPPC (shown in Fig. (4), together with higher scintillator quality, has demonstrated significant improvements. Figure 22 shows the MIP response of

\footnotetext{
${ }^{\text {e }}$ This is after correcting for known differences in the 2007 production due to absence of a photon screen and use
} of WLS fibre rather than direct coupling to the MPPC. 
a channel as a function of the distance from the MPPC, and the distribution of the ratio of response at the far end side to the MPPC side for all channels in the prototype (with the exception of four dead channels). A response ratio for each channel was determined from the result of a single exponential function: the measured position dependence was fitted by a single exponential function, and a response ratio defined as the ratio of this function at the two strip ends. The mean and RMS of the measured uniformity are $(88.3 \pm 4.3) \%$. This uniformity of the response within each strip has been measured using muon events by reconstructing the position within a strip using information from layers with different orientation. Simulation studies with and without a description of this nonuniformity demonstrated that the maximum degradation of the energy resolution due to non-uniformity is $\Delta\left(\sigma_{E} / E\right)=+0.04 \%$ at $2 \mathrm{GeV}$. Details of the simulation study are given in Section 6.1. Within uncertainties, there is no significant change of $\sigma_{E} / E$ as a function of beam position associated with the non-uniformity.
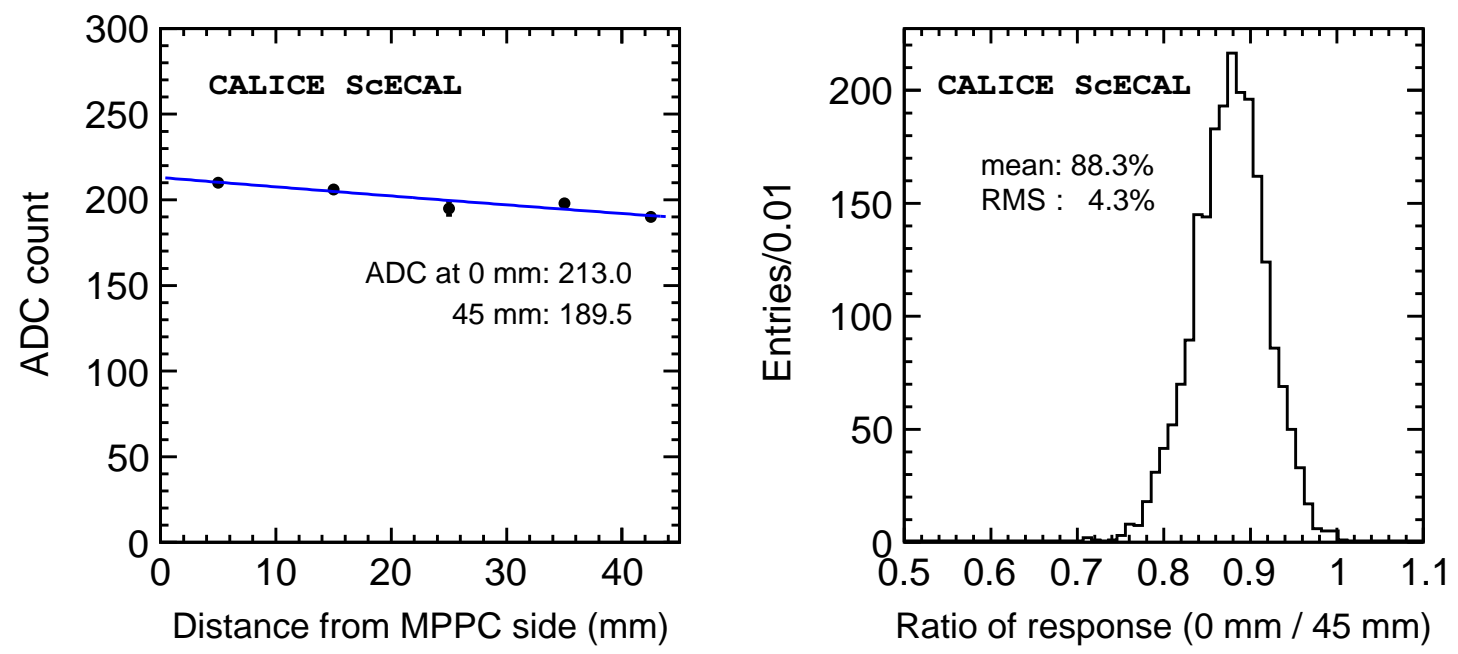

Figure 22: An example of the MIP response as a function of the distance from the MPPC side (left), and the distribution of the ratio of response at the far end side to the MPPC side, determined by fitting with an exponential function (right).

\section{Beam energy spread}

The beam provided at MT6 has a relative beam energy spread $\Delta E / E=2 \%$ for beam energies in the range $1-60 \mathrm{GeV}[28$. Tests of a $\mathrm{Pb} /$ glass calorimeter performed at the same beam-line led to an estimate of the relative beam energy spread of $2.7 \pm 0.3 \%$ for beam energies in the range 1-4 GeV [29]. Another experiment measured a relative spread of $2.3 \%$ for $8 \mathrm{GeV}[30$ by using the same $\mathrm{Pb} /$ glass calorimeter. A third study has also estimated a energy spread of $2.3 \%$ in the range $1.5-3.5 \mathrm{GeV}$ 31. Using these measurements we assign a beam energy spread of $(2.7 \pm 0.3) \%$ for beam energies between 2 and $4 \mathrm{GeV}$, and $(2.3 \pm 0.3) \%$ for the range $8-32 \mathrm{GeV}$. To estimate the intrinsic energy resolution of the prototype, this energy spread should be quadratically subtracted from the energy resolution determined. The systematic uncertainty on this procedure arises from the uncertainty of the intrinsic beam energy spread, taken to be $0.3 \%$, and is motivated by the spread and uncertainties of the available measurements.

\section{Summary of uncertainties on each beam energy}

Table 4 summarises the different systematic uncertainties for the considered beam energies together with the statistical uncertainties. Figure 23 shows the same data as those of Fig. 21, but with systematic uncertainties discussed above included. The systematic 
uncertainties have a size comparable with the run-to-run variations, except for the 12, 15, and $20 \mathrm{GeV}$ cases, where the variation is larger than the estimated uncertainties. Those data were acquired early in the test beam period when there were frequent changes made to the beam conditions. This potentially results in changes of the beam energy with changing beam conditions. We conservatively assign the SD of the observed run-to-run variations as the systematic uncertainties in such cases. Table 5 lists the sum of the individually estimated uncertainties and the deviations estimated from the run-to-run variation. To reduce the impact of double counting of uncertainties, the larger of the two values is assigned as the final systematic uncertainty for each individual beam energy. Table 6 lists the energy resolution at each beam energy after subtraction of the beam energy spread, together with its systematic and statistical uncertainties. The quadrature sum of all systematic effects is completely dominated by the beam energy spread.

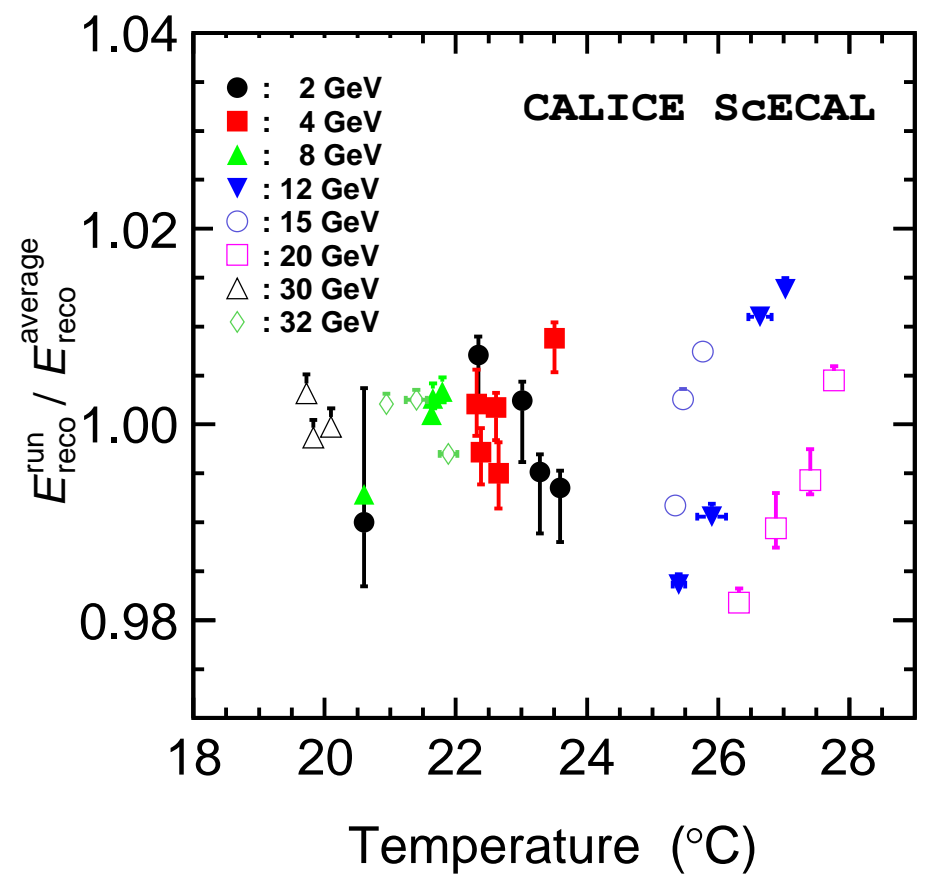

Figure 23: Ratio of the reconstructed mean of individual runs to their average vs. temperature.

\subsection{Linearity and energy resolution of the ScECAL prototype}

Figure 24 shows the mean reconstructed energy (as shown in Table 3, with uncertainties from Table 5) as a function of the incident beam energy. The solid line is the result of a linear fit to these measurements. The slope and offset are $(130.03 \pm 0.24) \mathrm{MIP} / \mathrm{GeV}$ and $(24.0 \pm 1.6)$ MIP, respectively. The figure also shows the deviation from linearity at each beam energy. The maximum deviation from linearity is $(1.1 \pm 0.4) \%$, at $8 \mathrm{GeV}$.

Figure 25 shows the energy resolution as a function of the inverse of the square root of the beam energy. The data points and their uncertainties are taken from Table 6; the intrinsic beam energy spread has been subtracted. The curve shows the result of a fit to the data using a two-component parametrisation of the energy resolution:

$$
\frac{\sigma_{E}}{E_{\text {reco }}}=\frac{C_{\text {stoch }}}{\sqrt{E_{\text {beam }}[\mathrm{GeV}]}} \oplus C_{\text {const }},
$$


Table 4: The uncertainties in the mean measured energy (\%) for combined data sets.

\begin{tabular}{cccccccc}
\hline \hline $\begin{array}{c}E_{\text {beam }} \\
{[\mathrm{GeV}]}\end{array}$ & range- $x$ & other cuts & $\left.c^{\mathrm{MIP}}\left(T_{0}=20^{\circ} \mathrm{C}\right)\right)$ & $\mathrm{d} c^{\mathrm{MIP}} / \mathrm{d} T$ & $N_{\text {pix }}$ & statistical & total \\
\hline 2 & ${ }_{-0.45}^{+0.22}$ & ${ }_{-0.37}^{+0.09}$ & \pm 0.23 & \pm 0.03 & \pm 0.11 & \pm 0.03 & ${ }_{-0.64}^{+0.36}$ \\
4 & ${ }_{-0.25}^{+0.21}$ & ${ }_{-0.22}^{+0.07}$ & \pm 0.09 & \pm 0.02 & \pm 0.01 & \pm 0.02 & ${ }_{-0.35}^{+0.24}$ \\
8 & ${ }_{-0.08}^{+0.12}$ & ${ }_{-0.03}^{+0.06}$ & \pm 0.21 & \pm 0.03 & \pm 0.05 & \pm 0.01 & ${ }_{-0.25}^{+0.27}$ \\
12 & ${ }_{-0.02}^{+0.10}$ & ${ }_{-0.04}^{+0.04}$ & \pm 0.16 & \pm 0.03 & \pm 0.05 & \pm 0.01 & ${ }_{-0.19}^{+0.21}$ \\
15 & ${ }_{-0.06}^{+0.07}$ & ${ }_{-0.03}^{+0.04}$ & \pm 0.13 & \pm 0.04 & \pm 0.04 & \pm 0.01 & ${ }_{-0.17}^{+0.18}$ \\
20 & ${ }_{-0.04}^{+0.18}$ & ${ }_{-0.04}^{+0.06}$ & \pm 0.13 & \pm 0.04 & \pm 0.04 & \pm 0.01 & ${ }_{-0.16}^{+0.24}$ \\
30 & ${ }_{-0.01}^{+0.13}$ & ${ }_{-0.02}^{+0.12}$ & \pm 0.12 & \pm 0.06 & \pm 0.16 & \pm 0.01 & ${ }_{-0.22}^{+0.28}$ \\
32 & ${ }_{-0.00}^{+0.02}$ & ${ }_{-0.03}^{+0.09}$ & \pm 0.23 & \pm 0.04 & \pm 0.13 & \pm 0.02 & ${ }_{-0.28}^{+0.30}$ \\
\hline
\end{tabular}

Table 5: Relative uncertainties of $E_{\text {reco }}$ from run-to-run variations (second column) and from all sources of estimated systematic uncertainties summed up in quadrature with statistical uncertainties (third column). The final relative uncertainties applied in the further linearity and resolution analysis are shown in the last column.

\begin{tabular}{|c|c|c|c|}
\hline \multirow{2}{*}{$\begin{array}{c}E_{\text {beam }} \\
{[\mathrm{GeV}]}\end{array}$} & \multicolumn{3}{|c|}{ Relative uncertainty (\%) } \\
\hline & Run variations & Estimated uncertainties & Final uncertainties \\
\hline 2 & \pm 0.58 & $-0.64+0.36$ & $-0.64+0.58$ \\
\hline 4 & \pm 0.34 & $-0.35+0.24$ & $-0.35+0.34$ \\
\hline 8 & \pm 0.44 & $-0.25+0.27$ & $-0.44+0.44$ \\
\hline 12 & \pm 1.23 & $-0.19+0.21$ & $-1.23+1.23$ \\
\hline 15 & \pm 0.66 & $-0.17+0.18$ & $-0.66+0.66$ \\
\hline 20 & \pm 0.79 & $-0.16+0.24$ & $-0.79+0.79$ \\
\hline 30 & \pm 0.17 & $-0.22+0.28$ & $-0.22+0.28$ \\
\hline 32 & \pm 0.27 & $-0.28+0.30$ & $-0.28+0.30$ \\
\hline
\end{tabular}


Table 6: Measured energy resolutions and their statistical and systematic uncertainties, after subtraction of beam energy spread, for each beam energy, $E_{\text {beam }}$.

\begin{tabular}{cccc}
\hline \hline $\begin{array}{c}E_{\text {beam }} \\
{[\mathrm{GeV}]}\end{array}$ & $\begin{array}{c}\text { energy resolution } \\
\sigma_{E} / E(\%)\end{array}$ & systematic & statistical \\
\hline 2 & 9.06 & \pm 0.34 & \pm 0.038 \\
4 & 6.25 & \pm 0.35 & \pm 0.028 \\
8 & 4.48 & \pm 0.33 & \pm 0.016 \\
12 & 3.72 & \pm 0.32 & \pm 0.018 \\
15 & 3.55 & \pm 0.31 & \pm 0.015 \\
20 & 3.04 & \pm 0.33 & \pm 0.030 \\
30 & 2.59 & \pm 0.34 & \pm 0.018 \\
32 & 2.52 & \pm 0.33 & \pm 0.022 \\
\hline
\end{tabular}

where $C_{\text {stoch }}$ and $C_{\text {const }}$ are free to vary in the fit and determined to be $(12.6 \pm 0.4) \%$ and $(1.1 \pm$ $0.4) \%$, respectively. The uncertainties include both systematic and statistical contributions.

The systematic uncertainties originating from the three calibration factors, $c^{\mathrm{MIP}}, c^{\mathrm{p} . e .}$, and $c^{\text {inter }}$ on the stochastic and constant terms of the energy resolution were investigated by using a pseudo-experiment method as discussed in Section [5.2. As examples, Fig. 26 shows the distribution of the stochastic (left) and constant (right) terms of the energy resolution in the pseudo-experiments in which $c^{\mathrm{MIP}}\left(T_{0}=20^{\circ} \mathrm{C}\right)$ was varied. The mean values slightly increased from the nominal value, because the random varies of those constants keep them away from true values. Therefore, we take RMS values of those for the uncertainty. The RMS of energy resolution for each beam energy are included in the systematic uncertainties in Table 6 as well as the uncertainty of $N_{\text {pix }}^{\text {eff }}$ and cut variations.

The statistical uncertainties in the energy resolution and stochastic terms of Equation 6 are determined by fitting to data, taking into account only statistical contributions from Table 6 . The central values of the stochastic term and the constant term are determined by using both statistical and systematic uncertainties in these fits.

The uncertainty arising from the intrinsic beam energy spread is considered to be completely correlated across all beam energies. The propagation of these uncertainties into the stochastic and constant terms are therefore conservatively estimated as the change from the nominal result caused by varying $C_{\text {stoch }}$ and $C_{\text {const }}$ coherently by $\pm 0.3 \%$ at all energies. These changes are taken to be the systematic uncertainties associated with these terms due to the beam energy spread, combined with the statistical uncertainty. Therefore, the residuals after quadratically subtracting statistical uncertainties from the uncertainties determined above, are considered as the systematic uncertainties from the beam energy spread. The uncertainty of the constant term from the intrinsic beam energy spread is $-0.7 \%,+0.5 \%$, while all other sources combined correspond to $\pm 0.09 \%$. The uncertainty assuming incoherent fluctuations is negligibly small.

Regarding the stochastic term, the uncertainties estimated above are much smaller than the case assuming the uncertainties of beam energy spread do not have coherent behaviour among energy points. Therefore, the systematic uncertainty originating from the uncertainties due to beam energy spread is conservatively adopted from the incoherent case as $0.4 \%$.

Therefore, the final results of the stochastic term and constant term can be expressed as:

$$
\begin{aligned}
& C_{\text {stoch }}=12.6 \pm 0.1 \text { (stat.) } \pm 0.4 \text { (syst.) } \% \mathrm{GeV}^{1 / 2} \\
& \left.C_{\text {const }}=1.1 \pm 0.1 \text { (stat. }\right)_{-0.7}^{+0.6} \text { (syst.) } \%
\end{aligned}
$$



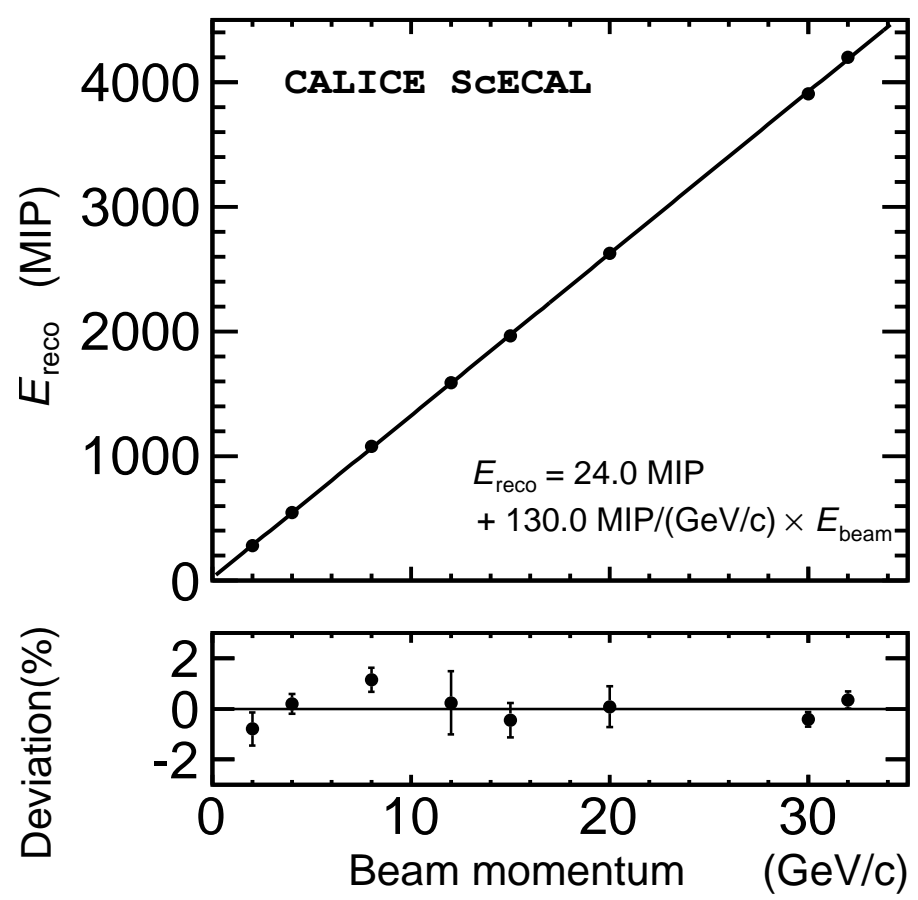

Figure 24: Response of the ScECAL prototype to 2-32 GeV electrons (top), deviation from the result of a linear fit divided by the linear fit (bottom). The error bars show the sum in quadrature of the statistical and systematic uncertainties.

\section{Comparison with Monte Carlo simulation}

\subsection{ScECAL prototype simulation}

The test beam setup was simulated using MoKka 32, a GEANT4 [33] based detector simulation framework. We selected a reference physics list of QGSP_BERT in the GEANT4 version 9.6 p1. The ScECAL simulation model consisted of 30 layers, each being composed of the absorber, a scintillator between two reflectors, readout instrumentation, and an air gap. The readout instrumentation layer was simulated as a uniform mixture of polyimide flat cable, clear fibre, polyvinyl chloride sheet, glass fibre and air. The scintillator layer was segmented in the same way as the prototype, but the reflectors between strips and the MPPC volumes were not simulated because the physical properties of those small materials are close to those of the scintillator. The absorber layers were made of a mixture of elements, as discussed in Section 2.1, with the measured density and mass fraction.

As the first step of simulation, $32 \mathrm{GeV}$ muon events were generated corresponding to each real run. From these simulated events, the energy deposited by a MIP, $E_{\mathrm{MIP}, i}^{\mathrm{dep}} / \mathrm{MIP}$, was determined as the MPV of the distribution of deposited energy in each channel. After determining $E_{\mathrm{MIP}, i}^{\mathrm{dep}} / \mathrm{MIP}$, each energy deposit was converted into the number of detected photons, p.e. $i, k$ using the following:

$$
\text { p.e. }{ }_{i, k}=e_{i, k}^{\text {dep }}\left(E_{\mathrm{MIP}, i}^{\mathrm{dep}} / \mathrm{MIP}\right)^{-1} R_{\mathrm{MIP} / \text { p.e. }}\left(T_{0}\right) \text {, }
$$

where $e_{i, k}^{\mathrm{dep}}$ is the energy deposited in channel $i$ in the event under consideration, $k$, and $R_{\mathrm{MIP} / \text { p.e. }}(T)=c_{i}^{\mathrm{MIP}}(T) /\left(c_{i}^{\text {p.e. }}(T) / c_{i}^{\text {inter }}\right)$ is taken from real data. This p.e. $i, k$ was then binomially fluctuated, thereby smearing the distribution of deposited energy in the number of p.e. for each channel 34. This smearing method-photon-statistics-smearing - was also applied to 


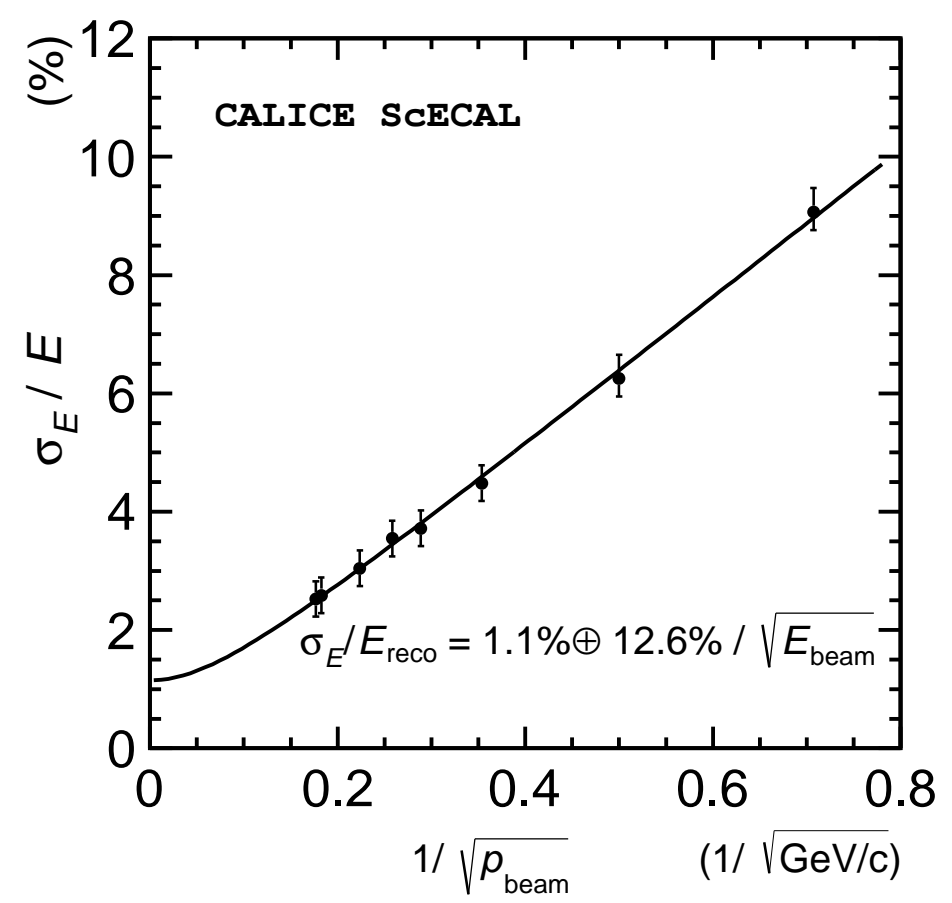

Figure 25: Energy resolution of the ScECAL as a function of the inverse square root of the beam energy. The error bars show the sum in quadrature of the statistical and systematic uncertainties.

all electron beam events in the simulation. From the MPV of the smeared distribution, an averaged $E_{\mathrm{MIP}}^{\mathrm{dep}} / \mathrm{MIP}$ of all channels was determined.

With this ratio, $E_{\mathrm{MIP}}^{\mathrm{dep}} / \mathrm{MIP}$, the digitisation procedure for each electron event is carried out as follows:

1. the deposited energy of each channel is converted into the equivalent number of MIPs: $n_{i, k}^{\mathrm{MIP}}=e_{i, k}^{\mathrm{dep}} /\left(E_{\mathrm{MIP}}^{\mathrm{dep}} / \mathrm{MIP}\right)$,

2. $n_{i, k}^{\mathrm{MIP}}$ is converted into the number of photoelectrons, $n_{i, k}^{\text {p.e. }}$, by multiplying by $R_{\mathrm{MIP} / \text { p.e. }}(T)$,

3. MPPC non-linear response is taken into account according to Equation 1 with $N_{\text {pix }}^{\text {eff }}$ yielding $n_{i, k}^{\mathrm{sat}}$,

4. Binomial fluctuations are applied to $n_{i, k}^{\text {sat }}$ to account for effects of photon statistics and finally this value is converted into the ADC counts, by multiplying by $c_{i}^{\text {p.e. }}(T) / c_{i}^{\text {inter }}$.

These digitised simulation data were analysed with the same computer code as the real data.

In this way, both the photon statistics and effects of non-linear response are taken into account for each channel of each event. The beam energy spread discussed in Section 5.2 was implemented as a Gaussian distribution and the geometrical beam spread in $x$ and $y$ were also taken into account using the distribution of the gravitational centre of energy in data. The material in the beam-line upstream of the prototype was simulated as three plastic scintillator trigger counters and one plastic scintillator veto counter. The (downstream) AHCAL and TCMT prototypes were not simulated, because they were used to remove muon and pion contaminations in data, whereas the simulated events did not include these contaminations, and the electron efficiency is almost unchanged.

Dead channels and detector noise were also implemented according to run-by-run detector conditions. The modelling of noise was carried out using the random trigger data introduced 

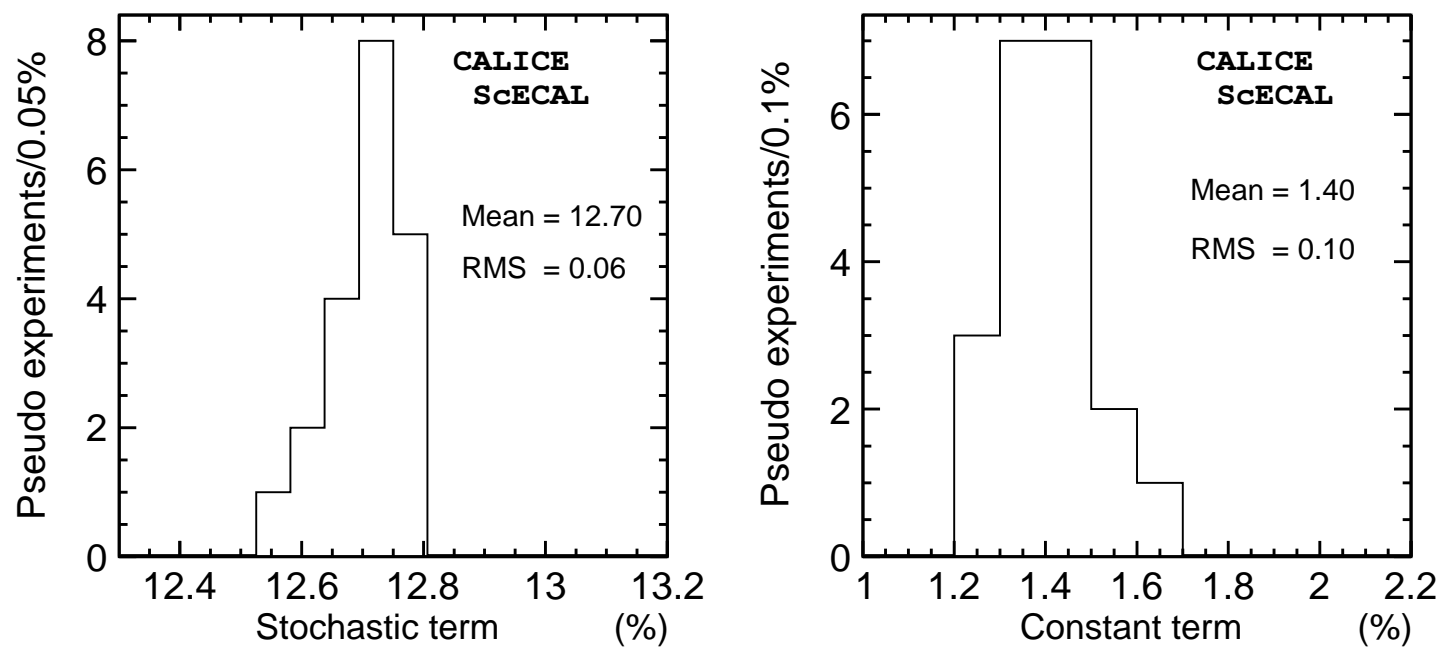

Figure 26: Distribution of the stochastic (left) and constant (right) terms of the energy resolution in 20 pseudo-experiments in which $c^{\mathrm{MIP}}\left(T_{0}=20^{\circ} \mathrm{C}\right)$ was varied.

in Section 4, allowing a noise signal to be overlaid onto each channel of each event: the noise signal of each channel in data is added to the simulated signal of the channel concerned in the digitisation procedure. The number of the random trigger events is between 5000 and 10000 per run. Therefore, the noise events were reused cyclically for the simulations of a given run.

\subsection{Shower profile}

It is essential for the simulation to accurately model the material composition of the detector. As mentioned in Section 2.1 the measured density of the absorber plates is $14.25 \pm 0.04 \mathrm{~g} / \mathrm{cm}^{3}$. This can be compared with the density calculated from the known constituents of the detector and their properties, giving a density of $14.76 \pm 0.13 \mathrm{~g} / \mathrm{cm}^{3}$ with $\rho_{\mathrm{WC}}=15.63 \pm 0.1 \mathrm{~g} / \mathrm{cm}^{3}\left[35, \rho_{\mathrm{Co}}=\right.$ $8.9 \mathrm{~g} / \mathrm{cm}^{3}$, and $\rho_{\mathrm{Cr}}=7.19 \mathrm{~g} / \mathrm{cm}^{3}$. This discrepancy requires a correction of the composition measured by EDX and X-ray diffraction, because the density by direct measurement is reliable.

We investigated two models for the correction: 1) weight ratio of Co to $\mathrm{WC}$ was changed to the direct measured density of the plate ("balanced" model), and 2) vacancies were uniformly distributed into the plate keeping the composition of materials ("vacancy" model). Details are explained in Appendix A.

Figure 27 shows comparisons of energy deposits on layers among both simulation cases and data. The best agreement is found using the balanced model, which agrees with data in the mean ratio, $0.98 \pm 0.04(\mathrm{SD})$ with a small slope of $-0.00064 \pm 0.00003$ /layer. Therefore, we use the "balanced" model in subsequent discussions. The systematic uncertainty from the model dependence is negligible; $-0.16 \pm 0.01$ on the mean response $\mathrm{dMIP} / \mathrm{d} E_{\text {beam }},+0.67 \pm 0.01 \mathrm{MIP}$ on the offset, $0.05 \pm 0.05 \%$ on the constant term of the energy resolution, and $+0.17 \pm 0.05 \%$ on the stochastic term.

Figure 28 shows comparisons of energy deposits projected on the $x$ axis in simulation and data. The simulations predict narrower lateral profiles than those observed in data in the "core" region (within $\pm 30 \mathrm{~mm}$ ), whereas the simulations have wider tails than the data. The origin of this discrepancy is as yet unexplained: we investigated the results of changing the detector angle with respect to the beam direction, the number of effective pixels, and the physics list to higher precision electromagnetic tracking [34, none of which was responsible for the effects observed. The narrower shower cores are not explained by the uncertainty of absorber composition, because we validated that the radiation length which determines the Molière radius of the detector was 


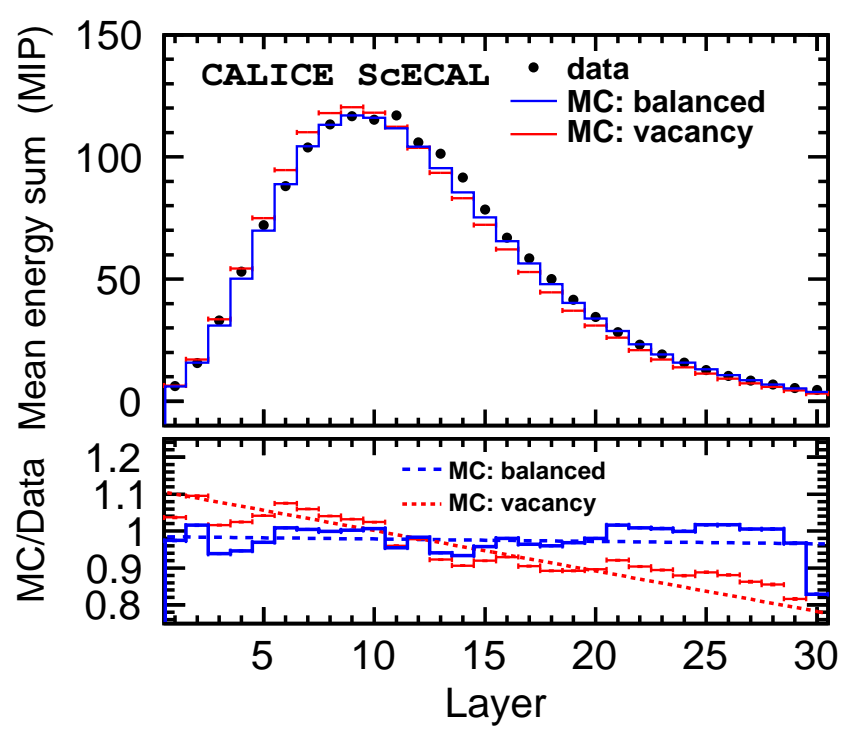

Figure 27: Comparison between data and Monte Carlo for the longitudinal energy deposition profile, using $12 \mathrm{GeV}$ electron beam data. MC to data ratio shows up to $10 \%$ discrepancy for the vacancy model. The balanced model which is the second composition of the absorber plates clearly improves agreement between data and MC. Dotted and dashed lines show linear fitting results.

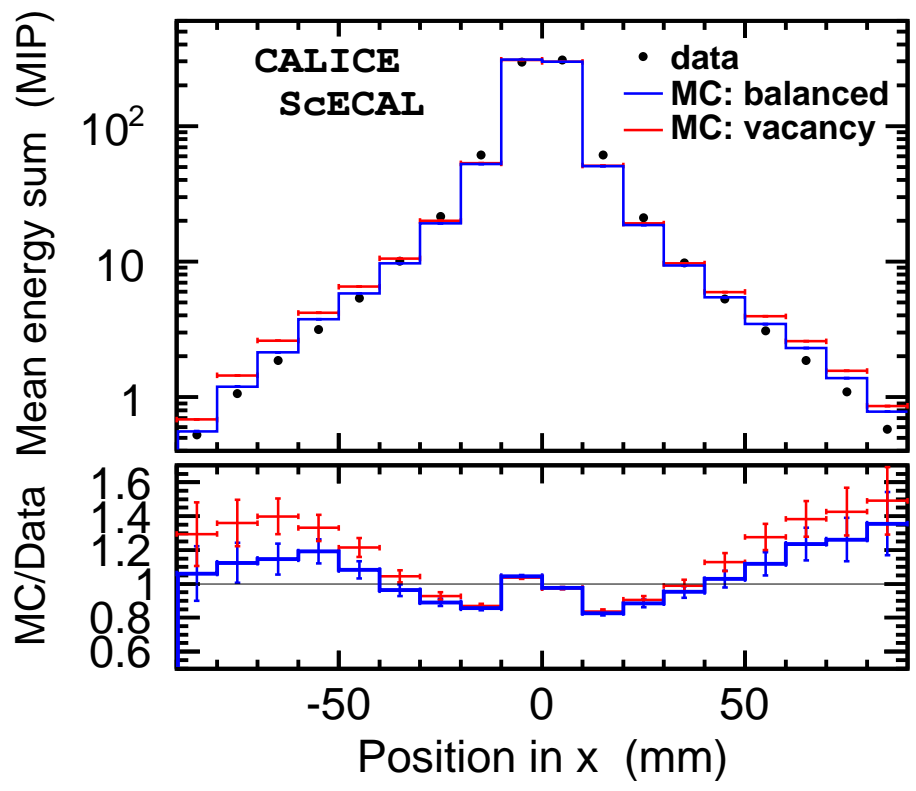

Figure 28: Comparison of the lateral energy deposition profile, using $12 \mathrm{GeV}$ electron beam data as an example. An energy sum is a collection of energy in the same lateral position on only those layers which have a $10 \mathrm{~mm}$ segmentation in the $x$ direction. The position is the distance from the energy centre of the cluster - centre of gravity - for each event. 
correct by agreement of longitudinal profiles between data and $\mathrm{MC}$ with the balanced absorber composition.

\subsection{Comparisons of linearity and resolution}

Figure 29 left compares the predicted response of the prototype to electrons with data. The slope observed in the simulation, $\mathrm{dMIP} / \mathrm{d} E_{\text {beam }}=130.27 \pm 0.06 \mathrm{MIP} / \mathrm{GeV}$, is consistent with that in the data of $130.03 \pm 0.24 \mathrm{MIP} / \mathrm{GeV}$, whereas the offset is $-3.0 \pm 0.1 \mathrm{MIP}$, some $27 \mathrm{MIP}$ smaller than found in data. This observation is illustrated clearly by the ratio of simulation to data in Fig. 29 left, bottom, suggesting the existence of a constant difference for all energies. This potentially originated by a small, residual background contamination in the data, dispite the detector noise is determined using random trigger events overlaid on the simulated events. The average difference of reconstructed energy between simulation and data is $-0.18 \pm 0.20$ (RMS) GeV.

Figures 29 right shows the energy resolution of data and simulation with several different conditions modelled in the simulations. The simulation described in Section 6 - denoted by gMC w/ detail factors" - agrees with the data, within uncertainties. The discrepancy persists even if the beam energy spread from higher beam energies is applied to the data recorded at 2 and $4 \mathrm{GeV}(2.3 \pm 0.3 \%)$. We discuss other MC models in Section 7
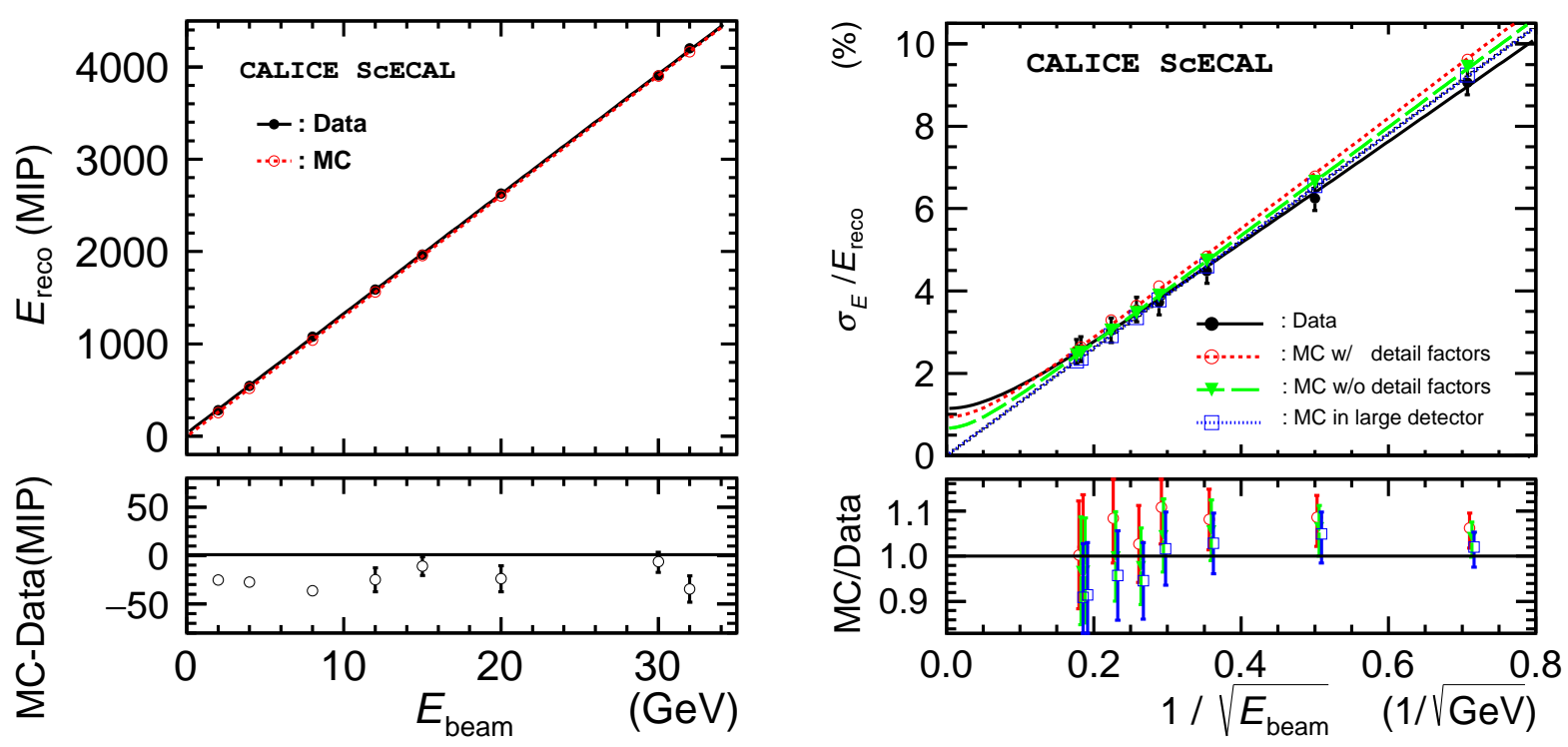

Figure 29: The response (left) and the energy resolution (right) of data and the simulated prototype to the electron beams. "detail factors" refers to the implementation of fine details, including photon statistics, effects of the MPPC non-linear response and the overlaying of noise. "large detector" refers to a simulation in which the dimensions of the detector have been increased by a factor of three, without the implementation of the "detail factors", to study the impact of leakage.

\section{Discussion}

The ScECAL prototype has shown a linear energy response for electron beam energies in the range between 2 and $32 \mathrm{GeV}$, with a maximum deviation from linearity of $(1.1 \pm 0.4) \%$ at $8 \mathrm{GeV}$. Although this experiment was performed in an environment subject to large variations of the ambient temperature, between $19^{\circ} \mathrm{C}$ and $27.5^{\circ} \mathrm{C}$, the calibration procedure, consisting of temperature-dependent of ADC-MIP and ADC-photoelectron conversion factors for each channel, successfully controlled the influence of these temperature variations. This gives clear 


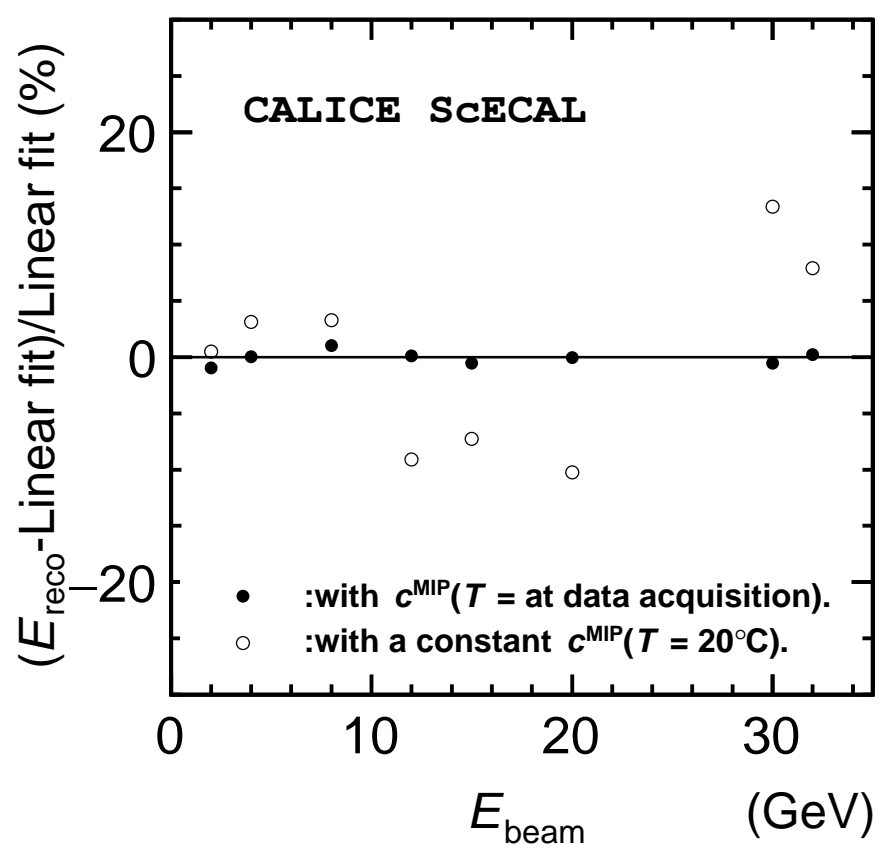

Figure 30: Deviations from linear energy response: the data were calibrated with the ADC-MIP and ADC-photoelectron conversion factors at $20^{\circ} \mathrm{C}$ (circles: only with statistical errors), and with the ADC-MIP and ADC-photoelectron conversion factors as functions of temperature (black dots: with statistical and systematic errors).

evidence that a SiPM-based scintillator tungsten ECAL can be used even in such serious temperature conditions. Figure 30 compares the deviations from linear energy response when using temperature-independent, without temperature correction, and temperature-dependent conversion factors, with temperature correction.

The variation of the $c^{\mathrm{MIP}}$ is $23 \%$ as shown in Fig. 15. This variation is larger than the expected value considering the variance in capacitance of the MPPCs used, as shown in Fig. 7 , and that the over-voltage of every channel was uniformly set to $3 \mathrm{~V}$. The most probable reason for this variation is a mis-alignment of the WLS fibre and MPPC positions: a lateral shift of the WLS fibre to the sensitive area of the MPPC decreases the photon yield of this scintillatorMPPC unit. This is caused by difficulties in the precise control of the position and size of the hole when using the extrusion method to manufacture the scintillator strips. Although the performance of the present prototype is sufficient, improved MPPC-fibre matching or direct coupling between the MPPC and scintillator have the potential to improve performance [36]. The CALICE Collaboration is currently studying $5 \mathrm{~mm}$-wide scintillator strips directly coupled to MPPCs [37.

The stochastic term in the energy resolution, determined as $(12.6 \pm 0.4) \% / \sqrt{E[\mathrm{GeV}]}$ for electron beam energies in the range $2-32 \mathrm{GeV}$, is significantly better than the requirement of $15 \% / \sqrt{E[\mathrm{GeV}]}$. This fact indicates that we can reduce the sampling ratio by reducing scintillator thickness. This is one of the advantages of the ScECAL that users can easily optimise the scintillator thickness to achieve a suitable performance. Actually, the CALICE Collaboration is currently developing the ScECAL with 1.5-2 mm thick scintillator strips 37.

The simulation provided a good description of the prototype data after the inclusion of a model of photon statistics, effect of the MPPC non-linear response and noise effects. The largest 
uncertainty in the input parameters for the simulation was the uncertainty of the intrinsic beam energy spread. The energy resolution of data and simulation are consistent when all of these uncertainties are taken into consideration.

Regarding the positive offset of the response corresponding to $0.18 \pm 0.20$ (RMS) GeV, $[23 \pm$ 26(RMS) MIP], the overlaying of noise on the MC events does not reproduce this phenomenon. The agreement of $\mathrm{d} E / \mathrm{dMIP}$ between data and simulation, and the behaviour of MC-data in Fig. 29 left, bottom, indicate that the effect is coherent among the incident energies so that the offset is not induced by possible deficiencies in the correction of the MPPC non-linear response: effect by deficiencies possibly increases with the deposited energy increase.

We studied what conditions contributed to the energy resolution by comparing data and MC modelling of several alternative sets of conditions. To extract the effect of energy leakage a study was performed using a simulation in which a detector of linear dimensions three times greater in each dimension $\left(900 \times 900 \mathrm{~mm}^{2} \times 90\right.$ layers $)$. Figure 31 shows the fraction of energy leakage perpendicular to the nominal beam direction (lateral leakage) and in depth (longitudinal leakage) of the ScECAL prototype, estimated by comparison of deposited energy between large detector and prototype size. The total leakage is between 2.3 and $3 \%$ at all measured energies: the lateral leakage ratio decreases with increasing energy and dominates below $20 \mathrm{GeV}$, while the longitudinal ratio increases with energy. The total deposited energy as the reference does not include the energy leaking out via the front face of the ScECAL.

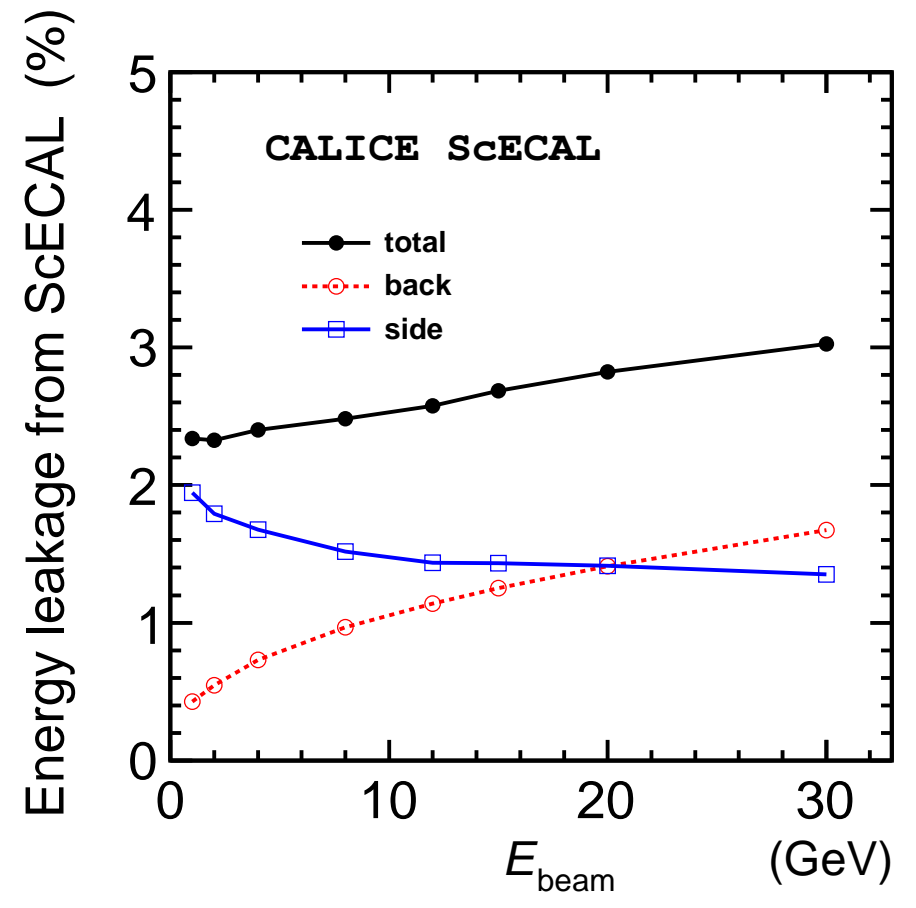

Figure 31: Relative leakage of the electron energy in the lateral (open boxes) and longitudinal (open circles) directions. The black markers show the total leakage.

Table 7 lists the energy resolution of data and simulation of such a large detector with several other modelings. Comparison of simulated results of the resolution parameters between large detector and the actual size of the detector shows that the leakage alone contributes half the uncertainty in the of constant term; increasing the constant term $\Delta\left(\sigma_{E} / E\right)=+0.66 \%$. The leakage also increases the stochastic term by a relative $1.8 \%$, which corresponds to a factor of 2.5 for the statistical uncertainty. 
Table 7: The constant term and the stochastic term of the energy resolution for data and the various simulations.

\begin{tabular}{|c|c|c|c|c|}
\hline data/MC & size(m;m;layers) & simulation details* & constant term $(\%)^{\dagger}$ & stochastic term $(\%)^{\dagger}$ \\
\hline $\mathrm{MC}$ & $0.9 \times 0.9 \times 90$ & without & $0.00 \pm 0.22$ & $13.03 \pm 0.04$ \\
\hline $\mathrm{MC}$ & $0.18 \times 0.18 \times 30$ layers & without & $0.66 \pm 0.08$ & $13.26 \pm 0.08$ \\
\hline $\mathrm{MC}$ & $0.18 \times 0.18 \times 30$ layers & with & $0.94 \pm 0.03$ & $13.58 \pm 0.04$ \\
\hline $\mathrm{MC}$ & $0.18 \times 0.18 \times 30$ layers & with $^{\ddagger}$ & $0.78 \pm 0.03$ & $13.52 \pm 0.03$ \\
\hline data & $0.18 \times 0.18 \times 30$ layers & - & $1.1 \pm 0.7$ & $12.6 \pm 0.4$ \\
\hline \multicolumn{5}{|c|}{$\begin{array}{l}\text { * Includes modelling of finite photon statistics, MPPC non-linear response, beam energy, position } \\
\text { fluctuation and noise; see Section } 6.1 \\
\text { † including systematic and statistical uncertainties for data; statistic only for MC. }\end{array}$} \\
\hline
\end{tabular}

Photon statistics, correction of the MPPC non-linear response, non-uniformity of single scintillator response and the noise have a combined contribution to the degradation of the energy resolution that is comparable to the effect of leakage. For details of these contributions, comparing simulation with and without these effects indicated that increase of the constant term is $\Delta\left(\sigma_{E} / E\right)=+0.67 \%$ whereas decrease of the stochastic term is relatively $2.4 \%$.

Similarly, a comparison of the impact of overlaying noise on the simulation indicated that overlaying the noise increases the value of the constant term of the energy resolution by $+0.5 \%$ whereas the effect on the stochastic term is negligible.

\section{Conclusion}

A prototype of a Scintillator-Tungsten ECAL, designed for a future linear collider experiment, was constructed and tested at Fermilab in May 2009. This represents the large scale application of novel SiPM (MPPC) sensors and is a feasibility study for the realisation of a highly granular calorimeter using this type of photodetector.

The response of the prototype to electron beams with energies between 2 and $32 \mathrm{GeV}$ was studied. Despite the large environmental temperature variation, $19^{\circ} \mathrm{C}-27.5^{\circ} \mathrm{C}$, a stable, linear response with a maximum deviation from linearity of $1.1 \%$ was verified with a standard temperature correction procedure. The intrinsic energy resolution performance obtained, $(12.6 \pm 0.1$ (stat.) \pm 0.4 (syst. $)) / \sqrt{E[\mathrm{GeV}]} \oplus\left(1.1 \pm 0.1\right.$ (stat.) ${ }_{-0.7}^{+0.6}$ (syst. $\left.)\right) \%$, is sufficient for the anticipated requirements of a future linear collider. Each scintillator strip has sufficient uniformity of response with $(88.3 \pm 4.3) \%$ of the further side of the SiPM.

Potential systematic uncertainties arising from a number of sources have been studied, including: the precision of the beam energy spread; event selection cuts; ADC-MIP, ADCphotoelectron and inter-calibration factors; and the effective number of MPPC pixels. The most important uncertainty in the energy resolution is due to the uncertainty of the beam energy spread, $0.3 \%$.

\section{Acknowledgement}

We gratefully acknowledge the Fermilab managements for their support and hospitality, and their accelerator staff for the reliable and efficient beam operation. This work was supported by the FWO, Belgium; by the Natural Sciences and Engineering Research Council of Canada; by the Ministry of Education, Youth and Sports of the Czech Republic; by the European Union's 
Horizon 2020 Research and Innovation programme under Grant Agreement 654168; by the European Commission within Framework Programme 7 Capacities, Grant Agreement 262025; by the Alexander von Humboldt Stiftung $(\mathrm{AvH})$, Germany; by the Bundesministerium für Bildung und Forschung (BMBF), Germany; by the Deutsche Forschungsgemeinschaft (DFG), Germany; by the Helmholtz-Gemeinschaft (HGF), Germany; by the I-CORE Program of the Planning and Budgeting Committee, Israel; by the Nella and Leon Benoziyo Center for High Energy Physics, Israel; by the Israeli Science Foundation, Israel; by the JSPS KAKENHI Grant-in-Aid for Scientific Research (B) No.17340071 and specially promoted research No. 223000002, Japan; by the National Research Foundation of Korea; by the Korea-EU cooperation programme of National Research Foundation of Korea, Grant Agreement 2014K1A3A7A03075053; by the Russian Ministry of Education and Science contracts 3.2989.2017 and 14.A12.31.0006; by the Spanish Ministry of Economy and Competitiveness FPA2014-53938-C3-R2 and Grant MDM-2015-0509; by the Science and Technology Facilities Council, UK; by the National Science Foundation of the United States of America; by the Nuclear Physics, Particle Physics, Astrophysics and Cosmology Initiative, a Laboratory Directed Research and Development program at the Pacific Northwest National Laboratory, USA.

\section{Appendix A Composition of the absorber plate}

As discussed in 6.2, there is a discrepancy of absorber density between its direct measurement and estimation from the composition of materials, as determined with EDX. Two plausible explanations behind this apparent discrepancy are the following:

"balanced" model: EDX results have potentially unknown systematic uncertainties; the WC material is too hard to provide sufficiently many samples at various locations in a plate, although the two samples used for tests showed no evidence of significant differences in their composition;

"vacancy" model: because the WC is a sintered material, produced by compressing a powder, the absorber plate is not entirely uniform, and has vacancies; back-scattered electron imaging shows that the absorber plate is an aggregate of WC grains.

In the "balanced" model, the ratio of mass of WC to Co and $\mathrm{Cr}$ was decreased keeping the ratio of $\mathrm{Co}$ and $\mathrm{Cr}$ In the "vacancy" model, the absolute mass quantity of material within the MC model was reduced, so that the relative composition was maintained and the absorber had the density obtained from direct measurement, meaning that the absorber material has vacancies. Table 8 lists the composition of the absorber in these two cases.

Table 8: Composition of the absorber plate in mass fraction (\%) measured with EDX (vacancy) and adjusted components, $\mathrm{WC}: \mathrm{Co}+\mathrm{Cr}$, to have the density measured directly (balanced).

\begin{tabular}{crr}
\hline \hline Component & \multicolumn{1}{c}{ vacancy } & \multicolumn{1}{c}{ balanced } \\
\hline $\mathrm{W}$ & $81.82 \pm 0.31$ & $74.43 \pm 0.30$ \\
$\mathrm{C}$ & $5.35 \pm 0.02$ & $4.86 \pm 0.02$ \\
$\mathrm{Co}$ & $12.39 \pm 0.47$ & $19.99 \pm 0.45$ \\
$\mathrm{Cr}$ & $0.45 \pm 0.47$ & $0.72 \pm 0.45$ \\
\hline
\end{tabular}

Although both models agree with data in the mean ratio of longitudinal profile, $0.98 \pm 0.04$ (SD) for "balanced" model and $0.96 \pm 0.07$ (SD) for "vacancy" model as we can see in Fig.27 the gradients from a linear fit to the ratios show clearly better agreement with the "balanced" model. 
The slope for the "balanced" ("vacancy") model is $-0.00064 \pm 0.00003 /$ layer $(-0.01043 \pm$ 0.00003 /layer).

\section{References}

[1] J.-C. Brient and H. Videau, eConf, vol.C010630, p. E3047 (2001), arXiv:hep-ex/0202004.

[2] M. Thomson, Nucl.Inst.and Meth., A611, 25 (2009).

[3] ILD Concept Group, The international Large Detector Letter of Intent (2009).

[4] G. Bondarenko et al., Nucl.Inst.and Meth., A442 187. (2000).

[5] P. Buzhan et al., ICFA Instrum. Bull. 23, 28 (2001).

[6] P. Buzhan et al., Nucl.Inst.and Meth., A504 48, (2003).

[7] CALICE Collaboration, C. Adloff et al., "Construction and Commissioning of the CALICE Analog Hadron Calorimeter Prototype", JINST, 5, P05004 (2010).

[8] E. Garutti, "Silicon photomultipliers for high energy physics detectors", Jinst, 6, C10003 (2011).

[9] T. Behnke et al. ILC Technical Design Report, Vol.4, arXiv:1306.6329 (2013).

[10] K. Kotera et al., Nucl.Inst.and Meth., A 789, 158-164 (2015).

[11] K. Francis et al., Nucl.Inst.and Meth., A 763, 278-289 (2014).

[12] CALICE Collaboration, C. Adloff et al., "Electromagnetic response of a highly granular hadronic calorimeter", JINST, 6, P04003 (2011).

[13] CALICE Collaboration, C. Adloff et al., "Hadronic energy resolution of a highly granular scintillator-steel calorimeter using software compensation techniques", JINST, 7, P09017 (2012).

[14] Sung Hyun Chang et al.,

[15] Hamamatsu Photonics KK, http://jp.hamamatsu.com/en/index.html JKPS, Vol.53, No. 6, 3178 (2008).

[16] KURARAY CO., LTD., http://www.kuraray.com

[17] KIMOTO CO., LTD., http://www.kimoto.co.jp/english/company/index.html

[18] http://www.3m.com/3M/en_US/company-us/all-3m-products/ /

3M-Enhanced-Specular-Reflector-3M-ESR?N=5002385+3293061534\&rt=rud

[19] Ivo Polak, IP ASCR Pargue, talk at ECFA meeting in Valencia (2007). http://www-hep2.fzu.cz/calice/files/ECFA_Valencia.Ivo_CMB_Devel_nov06.pdf

[20] http://omega.in2p3.fr

[21] S. Blin, et al., LC-DET-2006-007.

[22] F. Gaede et al., LC-TOOL-2003-053, arXiv:physics/0306114

[23] L. Mineev et al. arXiv:1110.2651,

[24] H. Oide et al., Proceedings of Photon Det 2007, Kobe, PoS(PD07), 008, (2007).

[25] K. Kotera, W. Choi, and T. Takeshita, arXiv:1510.01102

[26] N. Feege, DESY-THESIS-11-048 (2010).

[27] CALICE Collaboration, CALICE Analysis note 006.

[28] C. Johnstone, Proc.,EPAC, p.p. 3242-3244, (2006), Edinburgh, Scotland.

[29] C. Polly, Special Reports of All Experimenters meetings FNAL, (2010), http://www.fnal.gov/directorate/program_planning/all_experimenters_meetings/special_reports/ Polly_T1005_08_23_10.pdf.

[30] T. Tsai, Special Reports of All Experimenters meetings FNAL, (2012) http://www.fnal.gov/directorate/program_planning/all_experimenters_meetings/special_reports/ Tsai_T1018_01_30_12.pdf.

[31] R. McNabb et al, Nucl.Inst.and Meth., A 602, 396-402 (2009).

[32] http://ilcsoft.desy.de/portal/software_packages/mokka/ 
[33] S. Agostinelli et al., Geant4-a simulation toolkit. Nucl.Inst.and Meth., A506, 250 (2003).

[34] O. Hartbrich, DESY-THESIS-2016-020 (2016).

[35] Haynes, William M., ed. (2011). CRC Handbook of Chemistry and Physics (92nd ed.). Boca Raton, FL: CRC Press. p. 4.96. ISBN 1439855110.

[36] F. Simon, C. Soldner, Nucl.Inst.and Meth., A620, 196-201 (2010).

[37] K. Kotera, IEEE, NSS/MIC conference record, (2014) Seattle, WA, USA, DOI: 10.1109/NSSMIC.2014.7431152. 